\title{
A NEW INFINITE GAME IN BANACH SPACES WITH APPLICATIONS
}

\author{
E. ODELL, TH. SCHLUMPRECHT, AND A. ZSÁK
}

\author{
Dedicated to Nigel J. Kalton on the occassion of his $60^{\text {th }}$ birthday.
}

\section{INTRODUCTION}

Let $X$ be a separable infinite-dimensional Banach space, and let $\mathcal{A}$ be a set of normalized sequences in $X$. We can consider a two-player game in $X$ each move of which consists of player S (subspace chooser) selecting some element $Y$ from the set $\operatorname{cof}(X)$ of finite-codimensional subspaces of $X$, and $\mathrm{P}$ (point chooser) responding by selecting a vector $y$ from the unit sphere $S_{Y}$ of $Y$. The game, which we shall refer to as the $\mathcal{A}$-game, consists of an infinite sequence of such moves generating a sequence $X_{1}, x_{1}, X_{2}, x_{2}, \ldots$, where $X_{i} \in \operatorname{cof}(X)$ and $x_{i} \in S_{X_{i}}$ for all $i \in \mathbb{N}$. S wins the $\mathcal{A}$-game if $\left(x_{i}\right)_{i=1}^{\infty} \in \mathcal{A}$.

Of course this game, which has its roots in the game described by W. T. Gowers [5] and in the notion of asymptotic structure [9], has certain limitations. Unlike the theory of asymptotic structure (where, for each $n \in \mathbb{N}$, a game is considered that consists of $n$ moves, where each move is the same as above), there is generally no unique smallest class $\mathcal{A}$ (depending on $X$ ) for which $\mathrm{S}$ has a winning strategy. However, one can hypothesize certain specific classes $\mathcal{A}$ for which $\mathrm{S}$ has a winning strategy for a given $X$ and deduce certain structural consequences. For example, if for some $K>0$ we let $\mathcal{A}$ be the class of sequences $K$-equivalent to the unit vector basis of $\ell_{p}(1<p<\infty)$, then any reflexive space $X$ in which $\mathrm{S}$ has a winning strategy for the $\mathcal{A}$-game in $X$, embeds into an $\ell_{p}$-sum of finite-dimensional spaces [10]. In fact, it was the problem of classifying subspaces of $\ell_{p}$-sums of finite-dimensional spaces that motivated the study of this game.

The general theme here is to take a coordinate-free property of a space $X$, recast it in terms of $\mathrm{S}$ having a winning strategy in the $\mathcal{A}$-game for a suitable class $\mathcal{A}$, and then to show that $X$ embeds into a space with an FDD (finite-dimensional decomposition) which has the "coordinatized" version of the property we started with. In addition to the $\ell_{p}$ result in [10] cited above this general theme was followed in [11] and [12. In [11] reflexive spaces $X$ were studied for which $\mathrm{S}$ has a winning strategy for both games corresponding to the classes $\mathcal{A}_{p}$ of normalized basic sequences with an $\ell_{p}$-lower estimate and $\mathcal{A}^{q}$ of normalized basic sequences with an $\ell_{q}$-upper estimate $(1<q \leq p<\infty)$. The end result was that $X$ embeds into a reflexive space with an FDD such that every block sequence satisfies $\ell_{p}$-lower and $\ell_{q}$-upper estimates. A consequence of this is that one can construct a separable, reflexive space universal for the class of separable, uniformly convex spaces or, more generally, for the class $C_{\omega}=\left\{X: X\right.$ is separable, reflexive, $\left.\mathrm{Sz}(X) \leq \omega, \mathrm{Sz}\left(X^{*}\right) \leq \omega\right\}$, where $\mathrm{Sz}(Y)$ denotes the Szlenk index of a separable Banach space $Y$. Recently an alternative proof of the universal result was given [3] using powerful set-theoretical notions (although, the FDD structural results cannot be obtained in this way). We should also note that a set-theoretical study of $\mathcal{A}$-games was given by C. Rosendal [14].

Date: October 25, 2018.

2000 Mathematics Subject Classification. Primary 46B20.

Research of the first two authors was supported by the National Science Foundation. 
The motivation behind this paper arose from a problem posed to us by A. Pełczyński. Given $\alpha<\omega_{1}$, does there exist a separable, reflexive space universal for the class $C_{\alpha}$ (defined as above with $\omega$ replaced by $\alpha$ ). Thus far the authors of [3] have been unable to extend their techniques to this problem. In researching Pełczyński's problem we discovered that it was necessary to consider a new game and solve the corresponding embedding problem in this context.

The game is played as follows. In each move of the game $\mathrm{S}$ (subspace chooser) selects $k \in \mathbb{N}$ and $Y \in \operatorname{cof}(X)$, and then $\mathrm{P}$ (point chooser) responds by choosing $y \in S_{Y}$. The game then consists of an infinite sequence of moves generating a sequence $k_{1}, X_{1}, x_{1}, k_{2}, X_{2}, x_{2}, \ldots$, where $k_{i} \in \mathbb{N}, X_{i} \in \operatorname{cof}(X)$ and $x_{i} \in S_{X_{i}}$ for all $i \in \mathbb{N}$. Given a normalized, 1-unconditional sequence $\left(v_{i}\right), \mathrm{S}$ is declared winner of this game if $\left(v_{k_{i}}\right)$ is dominated by $\left(x_{i}\right)$. We also consider, for given normalized 1unconditional sequence $\left(u_{i}\right)$, the version where $\mathrm{S}$ wins the game if $\left(x_{i}\right)$ is dominated by $\left(u_{k_{i}}\right)$. In the case where $\left(v_{i}\right)$ and $\left(u_{i}\right)$ are the unit vector bases of $\ell_{p}$ and $\ell_{q}$, respectively, this conforms to the games considered in [11, but Pełczyński's problem requires us to consider sequences $\left(v_{i}\right)$ and $\left(u_{i}\right)$ that are not subsymmetric. (In [12] in order to solve the problem of embedding an asymptotic $\ell_{p}$ space into one with an asymptotic $\ell_{p}$ FDD it was necessary to extend the results of [11] concerning $\ell_{p^{-}}$ lower and $\ell_{q}$-upper estimates to more general $\left(v_{i}\right)$-lower and $\left(u_{i}\right)$-upper estimates, but the game played did not change.)

The main results of this paper are given in Section 4. Theorems 12 and 15, and Corollary 13. In brief these theorems say the following. Suppose we are given normalized, 1-unconditional bases $\left(v_{i}\right)$ and $\left(u_{i}\right)$ with certain properties, and a reflexive space $X$. Assume that $\mathrm{S}$ wins the subsequential $\left(v_{i}\right)$-lower and the subsequential $\left(u_{i}\right)$-upper games described above. Then $X$ embeds into a space $Z$ with an FDD such that every block sequence satisfies subsequential $\left(v_{i}\right)$-lower and $\left(u_{i}\right)$-upper estimates. (Precise definitions of these estimates will be given below.)

One application of these theorems is a new proof of the results of [11. The application to the Pełczyński problem will appear in [13, where further machinery is necessary to exploit the results obtained here.

In Section 5 we derive some universal space consequences of our embedding theorems. Section 2 introduces our terminology, in particular we give precise definitions of various lower and upper norm estimates. Section 2 also contains some straightforward duality results concerning such norm estimates, and a combinatorial result (Proposition 5) that is key to embedding spaces satisfying the coordinate-free version of a certain property into a space with an FDD satisfying the "coordinatized" version of the same property.

In Section 3 we define the space $Z^{V}(E)$, where $Z$ is a Banach space with an FDD $E=\left(E_{i}\right)$ and $V$ is the closed linear span of a normalized, 1-unconditional sequence $\left(v_{i}\right)$. We develop the properties of $Z^{V}(E)$, in particular proving that, under appropriate hypotheses, $Z^{V}(E)$ is a reflexive space admitting subsequential $V$-lower estimates.

\section{DEFINITIONS AND PRELIMINARY RESULTS}

We begin with fixing some terminology. Let $Z$ be a Banach space with an FDD $E=\left(E_{n}\right)$. For $n \in \mathbb{N}$ we denote by $P_{n}^{E}$ the $n$-th coordinate projection, i.e., $P_{n}^{E}: Z \rightarrow E_{n}$ is the map defined by $\sum_{i} z_{i} \mapsto z_{n}$, where $z_{i} \in E_{i}$ for all $i \in \mathbb{N}$. For a finite set $A \subset \mathbb{N}$ we put $P_{A}^{E}=\sum_{n \in A} P_{n}^{E}$. The projection constant $K(E, Z)$ of $\left(E_{n}\right)$ (in $Z$ ) is defined by

$$
K=K(E, Z)=\sup _{m<n}\left\|P_{[m, n]}^{E}\right\|
$$


where $[m, n]$ denotes the interval $\{m, m+1, \ldots, n\}$ in $\mathbb{N}$. Recall that $K$ is always finite and, as in the case of bases, we say that $\left(E_{n}\right)$ is bimonotone (in $Z$ ) if $K=1$. By passing to the equivalent norm

$$
\|\mid \cdot\|: Z \rightarrow \mathbb{R}, \quad z \mapsto \sup _{m<n}\left\|P_{[m, n]}^{E}(z)\right\|,
$$

we can always renorm $Z$ so that $K=1$.

For a sequence $\left(E_{i}\right)$ of finite-dimensional spaces we define the vector space

$$
\mathrm{c}_{00}\left(\oplus_{i=1}^{\infty} E_{i}\right)=\left\{\left(z_{i}\right): z_{i} \in E_{i} \text { for all } i \in \mathbb{N} \text {, and }\left\{i \in \mathbb{N}: z_{i} \neq 0\right\} \text { is finite }\right\},
$$

which is dense in each Banach space for which $\left(E_{i}\right)$ is an FDD. For a set $A \subset \mathbb{N}$ we denote by $\bigoplus_{i \in A} E_{i}$ the linear subspace of $c_{00}\left(\oplus E_{i}\right)$ generated by the elements of $\bigcup_{i \in A} E_{i}$. As usual we denote the vector space of sequences in $\mathbb{R}$ which are eventually zero by $\mathrm{c}_{00}$. We sometimes will consider for the same sequence $\left(E_{i}\right)$ of finite-dimensional spaces different norms on $c_{00}\left(\oplus E_{i}\right)$. In order to avoid confusion we will therefore often index the norm by the Banach space whose norm we are using, i.e., $\|\cdot\|_{Z}$ denotes the norm of the Banach space $Z$.

If $Z$ has an FDD $\left(E_{i}\right)$, the vector space $c_{00}\left(\oplus_{i=1}^{\infty} E_{i}^{*}\right)$, where $E_{i}^{*}$ is the dual space of $E_{i}$ for each $i \in \mathbb{N}$, can be identified in a natural way with a $w^{*}$-dense subspace of $Z^{*}$. Note however that the embedding $E_{i}^{*} \hookrightarrow Z^{*}$ is, in general, not isometric unless $K=1$. We will always consider $E_{i}^{*}$ with the norm it inherits from $Z^{*}$ instead of the norm it has as the dual space of $E_{i}$. We denote the norm closure of $\mathrm{c}_{00}\left(\oplus_{i=1}^{\infty} E_{i}^{*}\right)$ in $Z^{*}$ by $Z^{(*)}$. Note that $Z^{(*)}$ is $w^{*}$-dense in $Z^{*}$, the unit ball $B_{Z^{(*)}}$ norms $Z$, and $\left(E_{i}^{*}\right)$ is an FDD of $Z^{(*)}$ having a projection constant not exceeding $K(E, Z)$. If $K(E, Z)=1$, then $B_{Z^{(*)}}$ is 1-norming for $Z$ and $Z^{(*)(*)}=Z$.

For $z \in \mathrm{c}_{00}\left(\oplus E_{i}\right)$ we define the support $\operatorname{supp}_{E}(z)$ of $z$ with respect to $\left(E_{i}\right)$ by

$$
\operatorname{supp}_{E}(z)=\left\{i \in \mathbb{N}: P_{i}^{E}(z) \neq 0\right\},
$$

and we define the range $\operatorname{ran}_{E}(z)$ of $Z$ with respect to $\left(E_{i}\right)$ to be the smallest interval in $\mathbb{N}$ containing $\operatorname{supp}_{E}(z)$. A sequence $\left(z_{i}\right)$ (finite or infinite) of non-zero vectors in $\mathrm{c}_{00}\left(\oplus E_{i}\right)$ is called a block sequence of $\left(E_{i}\right)$ if

$$
\max \operatorname{supp}_{E}\left(z_{n}\right)<\min \operatorname{supp}_{E}\left(z_{n+1}\right) \quad \text { whenever } n \in \mathbb{N}\left(\text { or } n<\operatorname{length}\left(z_{i}\right)\right) \text {. }
$$

A block sequence $\left(z_{i}\right)$ of $\left(E_{i}\right)$ is called normalized (in $Z$ ) if $\left\|z_{i}\right\|_{Z}=1$ for all $i \in \mathbb{N}$.

Let $\bar{\delta}=\left(\delta_{i}\right) \subset(0,1)$ with $\delta_{i} \downarrow 0$. A (finite or infinite) sequence $\left(z_{i}\right)$ in $S_{Z}$ is called a $\bar{\delta}$-skipped block sequence of $\left(E_{i}\right)$ if there exists a sequence $1 \leq k_{0}<k_{1}<k_{2}<\ldots$ in $\mathbb{N}$ such that

$$
\left.\left\|z_{n}-P_{\left(k_{n-1}, k_{n}\right)}^{E}\left(z_{n}\right)\right\|<\delta_{n} \quad \text { for all } n \in \mathbb{N} \text { (or } n \leq \operatorname{length}\left(z_{i}\right)\right) .
$$

Remark. A sequence $\left(F_{i}\right)$ of finite-dimensional spaces is called a blocking of $\left(E_{i}\right)$ if for some sequence $m_{1}<m_{2}<\ldots$ in $\mathbb{N}$ we have $F_{n}=\bigoplus_{j=m_{n-1}+1}^{m_{n}} E_{j}$ for all $n \in \mathbb{N}$ $\left(m_{0}=0\right)$. If $\left(F_{i}\right)$ is a blocking of $\left(E_{i}\right)$, and if $\left(x_{i}\right)$ is a $\bar{\delta}$-skipped block sequence of $\left(F_{i}\right)$, then $\left(x_{i}\right)$ is not necessarily a $\bar{\delta}$-skipped block sequence of $\left(E_{i}\right)$ (since in the definition of skipped block sequence we skip exactly one coordinate). Nevertheless it is clear that $\left(x_{i}\right)$ is a $2 K \bar{\delta}$-skipped block sequence of $\left(E_{i}\right)$, where $K$ is the projection constant of $\left(E_{i}\right)$ in $Z$.

Definition. Given two sequences $\left(e_{i}\right)$ and $\left(f_{i}\right)$ in some Banach spaces, and given a constant $C>0$, we say that $\left(f_{i}\right) C$-dominates $\left(e_{i}\right)$, or that $\left(e_{i}\right)$ is $C$-dominated by $\left(f_{i}\right)$, if

$$
\left\|\sum a_{i} e_{i}\right\| \leq C\left\|\sum a_{i} f_{i}\right\| \quad \text { for all }\left(a_{i}\right) \in \mathrm{c}_{00} \text {. }
$$

We say that $\left(f_{i}\right)$ dominates $\left(e_{i}\right)$, or that $\left(e_{i}\right)$ is dominated by $\left(f_{i}\right)$, if there exists a constant $C>0$ such that $\left(f_{i}\right) C$-dominates $\left(e_{i}\right)$. 
We shall now introduce certain lower and upper norm estimates for FDD's.

Definition. Let $Z$ be a Banach space with an FDD $\left(E_{n}\right)$, let $V$ be a Banach space with a normalized, 1 -unconditional basis $\left(v_{i}\right)$ and let $1 \leq C<\infty$.

We say that $\left(E_{n}\right)$ satisfies subsequential $C$-V-lower estimates (in $Z$ ) if every normalized block sequence $\left(z_{i}\right)$ of $\left(E_{n}\right)$ in $Z C$-dominates $\left(v_{m_{i}}\right)$, where $m_{i}=$ $\operatorname{minsupp}_{E}\left(z_{i}\right)$ for all $i \in \mathbb{N}$, and $\left(E_{n}\right)$ satisfies subsequential $C$-V-upper estimates (in $Z$ ) if every normalized block sequence $\left(z_{i}\right)$ of $\left(E_{n}\right)$ in $Z$ is $C$-dominated by $\left(v_{m_{i}}\right)$, where $m_{i}=\min \operatorname{supp}_{E}\left(z_{i}\right)$ for all $i \in \mathbb{N}$.

If $U$ is another space with a normalized and 1-unconditional basis $\left(u_{i}\right)$, we say that $\left(E_{n}\right)$ satisfies subsequential $C-(V, U)$ estimates $($ in $Z)$ if it satisfies subsequential $C$ - $V$-lower and $C$ - $U$-upper estimates in $Z$.

We say that $\left(E_{n}\right)$ satisfies subsequential $V$-lower, $U$-upper or $(V, U)$ estimates (in $Z$ ) if for some $C \geq 1$ it satisfies subsequential $C$ - $V$-lower, $C$ - $U$-upper or $C$ - $(V, U)$ estimates in $Z$, respectively.

Remark. Assume that $\left(E_{n}\right)$ satisfies subsequential $C$ - $V$-lower estimates in $Z$ and that $\left(z_{i}\right)$ is a normalized block sequence of $\left(E_{n}\right)$. If $\max _{\operatorname{supp}_{E}}\left(z_{i-1}\right)<m_{i} \leq$ $\min \operatorname{supp}_{E}\left(z_{i}\right)$ for all $i \in \mathbb{N}\left(\right.$ where $\left.\max \operatorname{supp}_{E}\left(z_{0}\right)=0\right)$, then $\left(z_{i}\right) C$-dominates $\left(v_{m_{i}}\right)$.

Another easy fact is that if every normalized block sequence $\left(z_{i}\right)$ of $\left(E_{n}\right)$ in $Z$ dominates $\left(v_{m_{i}}\right)$, where $m_{i}=\min _{\operatorname{supp}_{E}}\left(z_{i}\right)$ for all $i \in \mathbb{N}$, then $\left(E_{n}\right)$ satisfies subsequential $V$-lower estimates in $Z$.

Analogous statements hold for upper estimates.

We shall need a coordinate-free version of subsequential lower and upper estimates. One way of defining this is reminiscent of the notion of asymptotic structure. Let $V$ be a Banach space with a normalized and 1-unconditional basis $\left(v_{i}\right)$, and let $C \in[1, \infty)$. Assume that we are given an infinite-dimensional Banach space $X$. We say that $X$ satisfies subsequential $C$-V-lower estimates (respectively, subsequential $C$-V-upper estimates) if

$$
\begin{array}{lll}
\exists k_{1} \in \mathbb{N} & \exists X_{1} \in \operatorname{cof}(X) & \forall x_{1} \in S_{X_{1}} \\
\exists k_{2} \in \mathbb{N} & \exists X_{2} \in \operatorname{cof}(X) & \forall x_{2} \in S_{X_{2}} \\
\exists k_{3} \in \mathbb{N} & \exists X_{3} \in \operatorname{cof}(X) & \forall x_{3} \in S_{X_{3}}
\end{array}
$$

such that $k_{1}<k_{2}<\ldots$ and $\left(v_{k_{i}}\right)$ is $C$-dominated by (respectively, $C$-dominates) $\left(x_{i}\right)$. If $U$ is another Banach space with a normalized, 1-unconditional basis $\left(u_{i}\right)$, then we say that $X$ satisfies subsequential $C$ - $(V, U)$ estimates if it satisfies subsequential $C$ - $V$-lower and $C$ - $U$-upper estimates. Finally, we say that the Banach space $X$ satisfies subsequential $V$-lower, $U$-upper or $(V, U)$ estimates if for some constant $C$ it satisfies subsequential $C$ - $V$-lower, $C$ - $U$-upper or $C$ - $(V, U)$ estimates, respectively.

The above definitions are given more formally in the language of games. Let us recall from the Introduction that in our games each move consists of $\mathrm{S}$ (subspace chooser) selecting $k \in \mathbb{N}$ and $Y \in \operatorname{cof}(X)$, and then $\mathrm{P}$ (point chooser) responding by choosing $y \in S_{Y}$. The game then consists of an infinite sequence of moves generating a sequence $k_{1}, X_{1}, x_{1}, k_{2}, X_{2}, x_{2}, \ldots$, where $k_{i} \in \mathbb{N}, X_{i} \in \operatorname{cof}(X)$ and $x_{i} \in S_{X_{i}}$ for all $i \in \mathbb{N}$. Player $\mathrm{S}$ wins the game if $\left(v_{k_{i}}\right)$ is $C$-dominated by $\left(x_{i}\right)$, otherwise $\mathrm{P}$ is declared the winner. The space $X$ then satisfies subsequential $C$ $V$-lower estimates if and only if $\mathrm{S}$ has a winning strategy, i.e., there is a function $\phi$ such that given sequences $\left(k_{i}\right)$ in $\mathbb{N},\left(X_{i}\right)$ in $\operatorname{cof}(X)$, and $\left(x_{i}\right)$ in $X$ such that $x_{i} \in S_{X_{i}}$ and $\left(k_{i}, X_{i}\right)=\phi\left(x_{1}, x_{2}, \ldots, x_{i-1}\right)$ for all $i \in \mathbb{N}$, then $k_{1}<k_{2}<\ldots$ and 
$\left(v_{k_{i}}\right)$ is $C$-dominated by $\left(x_{i}\right)$. The notions of subsequential $C$ - $V$-upper estimates, subsequential $C-(V, U)$ estimates, etc. can be formalized in a similar way.

Yet another way of expressing subsequential lower and upper estimates in a coordinate-free way uses infinite, countably branching trees (see Proposition 1 below). This is not surprising since a winning strategy in the game described above corresponds naturally to such a tree. We define for $\ell \in \mathbb{N}$

$$
T_{\ell}=\left\{\left(n_{1}, n_{2}, \ldots, n_{\ell}\right): n_{1}<n_{2}<\ldots<n_{\ell} \text { are in } \mathbb{N}\right\}
$$

and

$$
T_{\infty}=\bigcup_{\ell=1}^{\infty} T_{\ell}, \quad T_{\infty}^{\text {even }}=\bigcup_{\ell=1}^{\infty} T_{2 \ell} .
$$

If $\alpha=\left(m_{1}, \ldots, m_{\ell}\right) \in T_{\ell}$, we call $\ell$ the length of $\alpha$ and denote it by $|\alpha|$, and $\beta=\left(n_{1}, \ldots, n_{k}\right) \in T_{\infty}$ is called an extension of $\alpha$, or $\alpha$ is called a restriction of $\beta$, if $\ell<k$ and $m_{i}=n_{i}$ for $i=1, \ldots, \ell$. We then write $\alpha<\beta$ and with this order both $T_{\infty}$ and $T_{\infty}^{\text {even }}$ are trees.

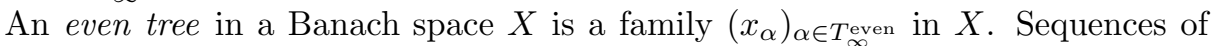
the form $\left(x_{(\alpha, n)}\right)_{n>n_{2 \ell-1}}$, where $\ell \in \mathbb{N}$ and $\alpha=\left(n_{1}, n_{2}, \ldots, n_{2 \ell-1}\right) \in T_{\infty}$, are called nodes of the tree. For a sequence $n_{1}<n_{2}<\ldots$ of positive integers the sequence $\left(x_{\left(n_{1}, n_{2}, \ldots, n_{2 \ell}\right)}\right)_{\ell=1}^{\infty}$ is called a branch of the tree.

If $\left(x_{\alpha}\right)_{\alpha \in T_{\infty}^{\text {even }}}$ is an even tree in a Banach space $X$ and if $T^{\prime} \subset T_{\infty}^{\text {even }}$ is closed under taking restrictions so that for each $\alpha \in T^{\prime} \cup\{\emptyset\}$ and for each $m \in \mathbb{N}$ the set $\left\{n \in \mathbb{N}:(\alpha, m, n) \in T^{\prime}\right\}$ is either empty or has infinite size, and moreover the latter occurs for infinitely many values of $m$, then we call $\left(x_{\alpha}\right)_{\alpha \in T^{\prime}}$ a full subtree

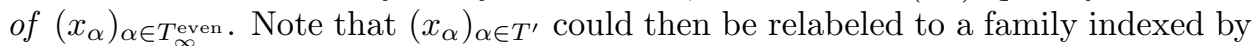
$T_{\infty}^{\text {even }}$, and note that the branches of $\left(x_{\alpha}\right)_{\alpha \in T^{\prime}}$ are branches of $\left(x_{\alpha}\right)_{\alpha \in T_{\infty}^{\text {even }}}$ and that the nodes of $\left(x_{\alpha}\right)_{\alpha \in T^{\prime}}$ are subsequences of certain nodes of $\left(x_{\alpha}\right)_{\alpha \in T_{\infty}^{\text {even }} \text {. }}$.

An even tree $\left(x_{\alpha}\right)_{\alpha \in T_{\infty}^{\text {even }}}$ in a Banach space $X$ is called normalized if $\left\|x_{\alpha}\right\|=1$ for all $\alpha \in T_{\infty}^{\text {even }}$, and is called weakly null if every node is a weakly null sequence. If $X$ has an FDD $\left(E_{n}\right)$, then $\left(x_{\alpha}\right)_{\alpha \in T_{\infty}^{\text {even }}}$ is called a block even tree of $\left(E_{n}\right)$ if every node is a block sequence of $\left(E_{n}\right)$.

Definition. Let $V$ be a Banach space with a normalized and 1-unconditional basis $\left(v_{i}\right)$, and let $C \in[1, \infty)$. Assume that we are given an infinite-dimensional Banach space $X$. We say that $X$ satisfies subsequential $C$ - $V$-lower tree estimates if every normalized, weakly null even tree $\left(x_{\alpha}\right)_{\alpha \in T_{\infty}^{\text {even }}}$ in $X$ has a branch $\left(x_{\left(n_{1}, n_{2}, \ldots, n_{2 i}\right)}\right)$ which $C$-dominates $\left(v_{n_{2 i-1}}\right)$.

We say that $X$ satisfies subsequential $C$-V-upper tree estimates if every normalized, weakly null even tree $\left(x_{\alpha}\right)_{\alpha \in T_{\infty}^{\text {even }}}$ in $X$ has a branch $\left(x_{\left(n_{1}, n_{2}, \ldots, n_{2 i}\right)}\right)$ which is $C$-dominated by $\left(v_{n_{2 i-1}}\right)$.

If $U$ is a second space with a 1 -unconditional and normalized basis $\left(u_{i}\right)$, we say that $X$ satisfies subsequential $C-(V, U)$ tree estimates if it satisfies subsequential $C$ - $V$-lower and $C$ - $U$-upper tree estimates.

We say that $X$ satisfies subsequential $V$-lower, $U$-upper or $(V, U)$ tree estimates if for some $1 \leq C<\infty X$ satisfies subsequential $C$ - $V$-lower, $C$ - $U$-upper or $C$ - $(V, U)$ tree estimates, respectively.

Remark. As in the FDD case, we do not need to fix a constant $C$ in the above definitions: if every normalized, weakly null even tree $\left(x_{\alpha}\right)_{\alpha \in T_{\infty}^{\text {even }}}$ in $X$ has a branch $\left(x_{\left(n_{1}, n_{2}, \ldots, n_{2 i}\right)}\right)$ which dominates $\left(v_{n_{2 i-1}}\right)$, then there exists a constant $C \geq 1$ such that $X$ satisfies subsequential $C$ - $V$-lower tree estimates. The analogous statement for upper estimates also holds. (See [12, Proposition 1.2].) 
Proposition 1 below shows that, under some mild hypotheses, the two coordinatefree versions of lower and upper estimates given above are essentially the same. Before stating this result we need a certain property of basic sequences defined in $[2]$.

Definition. Let $V$ be a Banach space with a normalized, 1-unconditional basis $\left(v_{i}\right)$ and let $1 \leq C<\infty$.

We say that $\left(v_{i}\right)$ is $C$-right-dominant (respectively, $C$-left-dominant) if for all sequences $m_{1}<m_{2}<\ldots$ and $n_{1}<n_{2}<\ldots$ of positive integers with $m_{i} \leq n_{i}$ for all $i \in \mathbb{N}$ we have that $\left(v_{m_{i}}\right)$ is $C$-dominated by (respectively, $C$-dominates) $\left(v_{n_{i}}\right)$. We say that $\left(v_{i}\right)$ is right-dominant or left-dominant if for some $C \geq 1$ it is $C$-right-dominant or $C$-left-dominant, respectively.

Remark. For $\left(v_{i}\right)$ to be right-dominant (respectively, left-dominant) it is enough to have the property that $\left(v_{m_{i}}\right)$ is dominated by (respectively, dominates) $\left(v_{n_{i}}\right)$ for all sequences $m_{1}<m_{2}<\ldots$ and $n_{1}<n_{2}<\ldots$ of positive integers with $m_{i} \leq n_{i}$ for all $i \in \mathbb{N}$. Also, $\left(v_{i}\right)$ is $C$-right-dominant (respectively, $C$-left-dominant) if and only if the sequence $\left(v_{i}^{*}\right)$ of biorthogonal functionals in $V^{(*)}$ is $C$-left-dominant (respectively, $C$-right-dominant).

Proposition 1. Let $V$ be a Banach space with a normalized and 1-unconditional basis $\left(v_{i}\right)$ and let $C, D \in[1, \infty)$. Let $X$ be an infinite-dimensional Banach space.

(a) Assume that $\left(v_{i}\right)$ is D-left-dominant. If $X$ satisfies subsequential $C$ - $V$-lower estimates, then for all $\varepsilon>0 X$ satisfies subsequential $(C D+\varepsilon)-V$-lower tree estimates.

(b) Assume that $X^{*}$ is separable. If $X$ satisfies subsequential $C$-V-lower tree estimates, then it also satisfies subsequential $C$-V-lower estimates.

Remark. Analogous results hold for upper estimates. For that in (a) we need to assume that $\left(v_{i}\right)$ is $D$-right-dominant.

Proof. (a) Assume that for some $\varepsilon>0$ there is a normalized, weakly null even tree $\left(x_{\alpha}\right)_{\alpha \in T_{\infty}^{\text {even }}}$ in $X$ such that for any sequence $n_{1}<n_{2}<\ldots$ of positive integers the branch $\left(x_{\left(n_{1}, n_{2}, \ldots, n_{2 i}\right)}\right)$ does not $(C D+\varepsilon)$-dominate $\left(v_{n_{2 i-1}}\right)$. We show that in this case $X$ does not satisfy subsequential $C$ - $V$-lower estimates by exhibiting a winning strategy for the point chooser P. Fix a sequence $\left(\delta_{i}\right) \subset(0,1)$ with $\Delta=\sum_{i} \delta_{i}$ satisfying

$$
C<\frac{C D+\varepsilon}{D} \cdot\left(1+\Delta \frac{C D+\varepsilon}{D}\right)^{-1} .
$$

Suppose the game starts with $\mathrm{S}$ picking $k_{1} \in \mathbb{N}$ and $X_{1} \in \operatorname{cof}(X)$. Since the nodes of $\left(x_{\alpha}\right)_{\alpha \in T_{\infty}^{\text {even }}}$ are weakly null, there exist $n_{1}, n_{2} \in \mathbb{N}$ such that $k_{1} \leq n_{1}<n_{2}$ and $d\left(x_{\left(n_{1}, n_{2}\right)}, X_{1}\right)<\delta_{1}$. P's response will be a $y_{1} \in S_{X_{1}}$ with $\left\|x_{\left(n_{1}, n_{2}\right)}-y_{1}\right\|<\delta_{1}$. In the second move $S$ picks $k_{2} \in \mathbb{N}$ and $X_{2} \in \operatorname{cof}(X)$. Then there exist $n_{3}, n_{4} \in \mathbb{N}$ such that $n_{2}<n_{3}<n_{4}, k_{2} \leq n_{3}$ and $d\left(x_{\left(n_{1}, n_{2}, n_{3}, n_{4}\right)}, X_{2}\right)<\delta_{2}$. P's response will be some $y_{2} \in S_{X_{2}}$ with $\left\|x_{\left(n_{1}, n_{2}, n_{3}, n_{4}\right)}-y_{2}\right\|<\delta_{2}$. In general, on the $j^{\text {th }}$ move of the game $(j \geq 2)$, S picks $k_{j} \in \mathbb{N}, X_{j} \in \operatorname{cof}(X)$. Then one can find $n_{2 j-1}, n_{2 j} \in \mathbb{N}$ such that $n_{2 j-2}<n_{2 j-1}<n_{2 j}, k_{j} \leq n_{2 j-1}$ and $d\left(x_{\left(n_{1}, n_{2}, \ldots, n_{2 j}\right)}, X_{j}\right)<\delta_{j}$. P's $j^{\text {th }}$ move will be some $y_{j} \in S_{X_{j}}$ such that $\left\|x_{\left(n_{1}, n_{2}, \ldots, n_{2 j}\right)}-y_{j}\right\|<\delta_{j}$.

Since the branch $\left(x_{\left(n_{1}, n_{2}, \ldots, n_{2 i}\right)}\right)$ does not $(C D+\varepsilon)$-dominate $\left(v_{n_{2 i-1}}\right)$, there exists $\left(a_{i}\right) \in \mathrm{c}_{00}$ such that

$$
\left\|\sum_{i} a_{i} v_{n_{2 i-1}}\right\|>(C D+\varepsilon)\left\|\sum_{i} a_{i} x_{\left(n_{1}, n_{2}, \ldots, n_{2 i}\right)}\right\| .
$$


We may assume without loss of generality that $\left\|\sum a_{i} v_{k_{i}}\right\|=1$. Using the $D$-leftdominant property of $\left(v_{i}\right)$ and that $k_{i} \leq n_{2 i-1}$ for all $i \in \mathbb{N}$, together with the choice of $\Delta$, an easy computation now gives

$$
\left\|\sum_{i} a_{i} v_{k_{i}}\right\|>C\left\|\sum_{i} a_{i} y_{i}\right\| .
$$

Thus $\mathrm{P}$ wins the game.

(b) Assume that $X$ does not satisfy subsequential $C$ - $V$-lower estimates. This means that $\mathrm{S}$ does not have a winning strategy, which in turn implies that there is a winning strategy $\phi$ for the point chooser (this follows from the fact that closed games [4] or, more generally, Borel games 8] are determined). Thus given sequences $\left(k_{i}\right)$ in $\mathbb{N},\left(X_{i}\right)$ in $\operatorname{cof}(X)$, and $\left(x_{i}\right)$ in $X$ such that $k_{1}<k_{2}<\ldots$ and $x_{n}=\phi\left(k_{1}, X_{1}, k_{2}, X_{2}, \ldots, k_{n}, X_{n}\right)$ for all $n \in \mathbb{N}$, then $x_{i} \in S_{X_{i}}$ for all $i \in \mathbb{N}$ and $\left(v_{k_{i}}\right)$ is not $C$-dominated by $\left(x_{i}\right)$. Fix a sequence $\left(X_{i}\right)$ of finite-codimensional subspaces of $X$ such that every bounded sequence $\left(x_{i}\right)$ with $x_{i} \in X_{i}$ for all $i \in \mathbb{N}$ is weakly null. This exists by the assumption that $X$ has separable dual.

We now construct a normalized, weakly null even tree in $X$ by recursion to show that $X$ does not satisfy subsequential $C$-V-lower tree estimates. For $\ell \in \mathbb{N}$ and $\alpha=\left(n_{1}, n_{2}, \ldots, n_{2 \ell}\right) \in T_{2 \ell}$ we set $x_{\alpha}=\phi\left(n_{1}, X_{n_{2}}, n_{3}, X_{n_{4}}, \ldots, n_{2 \ell-1}, X_{n_{2 \ell}}\right)$. It is easy to verify that $\left(x_{\alpha}\right)_{\alpha \in T_{\infty}^{\text {even }}}$ is a normalized, weakly null even tree in $X$, and that for any sequence $n_{1}<n_{2}<\ldots$ of positive integers the branch $\left(x_{\left(n_{1}, n_{2}, \ldots, n_{2 i}\right)}\right)$ does not $C$-dominate $\left(v_{n_{2 i-1}}\right)$.

If $V$ is a Banach space with a normalized, 1-unconditional basis, and if $N$ is an infinite subset of $\mathbb{N}$, we write $V_{N}$ for the closed linear span of $\left\{v_{i}: i \in N\right\}$. When we talk about subsequential $V_{N}$-lower estimates, etc., it will be with respect to the normalized, 1-unconditional basis $\left(v_{i}\right)_{i \in N}$ of $V_{N}$. We shall also write $\mathbb{N}^{(\omega)}$ for the set of all infinite subsets of $\mathbb{N}$.

Note that if $V$ is a Banach space with a normalized, 1-unconditional, leftdominant basis $\left(v_{i}\right)$, then for any space $Z$ with an FDD $\left(E_{i}\right)$ satisfying subsequential $V$-lower estimates in $Z$, the FDD $\left(E_{i}\right)$ will also satisfy subsequential $V_{N}$-lower estimates for any $N \in \mathbb{N}^{(\omega)}$. Later on we shall need a result that allows us to pass from subsequential $V_{N}$-lower estimates for some $N \in \mathbb{N}^{(\omega)}$ to subsequential $V$-lower estimates. Before stating this result we need a definition.

Definition. Let $V$ be a Banach space with a normalized, 1-unconditional basis $\left(v_{i}\right)$ and let $1 \leq C<\infty$.

We say that $\left(v_{i}\right)$ is $C$-block-stable if any two normalized block bases $\left(x_{i}\right)$ and $\left(y_{i}\right)$ with

$$
\max \left(\operatorname{supp}\left(x_{i}\right) \cup \operatorname{supp}\left(y_{i}\right)\right)<\min \left(\operatorname{supp}\left(x_{i+1}\right) \cup \operatorname{supp}\left(y_{i+1}\right)\right) \quad \text { for all } i \in \mathbb{N}
$$

are $C$-equivalent. We say that $\left(v_{i}\right)$ is block-stable if it is $C$-block-stable for some constant $C$.

Remark. It is routine to check that $\left(v_{i}\right)$ is $C$-block-stable if and only if the sequence $\left(v_{i}^{*}\right)$ of biorthogonal functionals in $V^{(*)}$ is $C$-block-stable.

A block-stable basis is a special case of a block-norm-determined FDD introduced by H. P. Rosenthal, who has initiated an exhaustive study of such FDDs [15].

Lemma 2. Let $V$ and $U$ be Banach spaces with normalized, 1-unconditional, blockstable bases $\left(v_{i}\right)$ and $\left(u_{i}\right)$, respectively, and assume that $\left(v_{i}\right)$ is dominated by $\left(u_{i}\right)$. Let $M \in \mathbb{N}^{(\omega)}$ and let $Z$ be a Banach space with an FDD $E=\left(E_{i}\right)$ satisfying subsequential $\left(V_{M}, U_{M}\right)$ estimates in $Z$. Then $W=Z \oplus_{\ell_{\infty}} V_{\mathbb{N} \backslash M}$ has an FDD $F=\left(F_{i}\right)$ satisfying subsequential $(V, U)$ estimates in $W$. 
Proof. Choose constants $B, C, D \in[1, \infty)$ such that $\left(v_{i}\right)$ and $\left(u_{i}\right)$ are $B$-block-stable, $\left(v_{i}\right)$ is $D$-dominated by $\left(u_{i}\right)$, and $\left(E_{i}\right)$ satisfies subsequential $C$ - $\left(V_{M}, U_{M}\right)$ estimates in $Z$. For each $n \in \mathbb{N}$ define

$$
F_{n}= \begin{cases}E_{i} & \text { if } n=m_{i} \text { for some } i \in \mathbb{N}, \\ \mathbb{R} \cdot v_{n} & \text { if } n \notin M .\end{cases}
$$

Then $F=\left(F_{n}\right)$ is an FDD for $W$ with projection constant $K(F, W) \leq K(E, Z)$. We now show that $\left(F_{i}\right)$ satisfies subsequential $\bar{C}$ - $(V, U)$ estimates in $W$, where $\bar{C}=B \cdot \max \{2 C, D\}$.

Let $\left(z_{i}\right)$ be a normalized block sequence of $\left(F_{n}\right)$ in $W$. For each $i \in \mathbb{N}$ let $k_{i}=\min \operatorname{supp}_{F}\left(z_{i}\right)$ and write

$$
z_{i}=z_{i}^{(1)}+z_{i}^{(2)}, \quad \text { where } z_{i}^{(1)} \in Z, z_{i}^{(2)} \in V_{\mathbb{N} \backslash M} .
$$

Fix $\left(a_{i}\right) \in \mathrm{c}_{00}$. We have

$$
\begin{aligned}
\left\|\sum_{i} a_{i} z_{i}^{(1)}\right\|_{Z} & \geq \frac{1}{C}\left\|\sum_{i} a_{i} \cdot\right\| z_{i}^{(1)}\left\|_{Z} \cdot v_{\operatorname{minsupp}}\left(z_{i}^{(1)}\right)\right\|_{V} \\
& \geq \frac{1}{B C}\left\|\sum_{i} a_{i} \cdot\right\| z_{i}^{(1)}\left\|_{Z} \cdot v_{k_{i}}\right\|_{V}
\end{aligned}
$$

and

$$
\left\|\sum_{i} a_{i} z_{i}^{(2)}\right\|_{V} \geq \frac{1}{B}\left\|\sum_{i} a_{i} \cdot\right\| z_{i}^{(2)}\left\|{ }_{Z} \cdot v_{k_{i}}\right\|_{V} .
$$

It follows that

$$
\begin{aligned}
\left\|\sum_{i} a_{i} z_{i}\right\|_{W} & \geq \frac{1}{B C} \max \left\{\left\|\sum_{i} a_{i} \cdot\right\| z_{i}^{(1)}\left\|_{Z} \cdot v_{k_{i}}\right\|_{V},\left\|\sum_{i} a_{i} \cdot\right\| z_{i}^{(2)}\left\|_{Z} \cdot v_{k_{i}}\right\|_{V}\right\} \\
& \geq \frac{1}{2 B C}\left\|\sum_{i} a_{i} v_{k_{i}}\right\|_{V} .
\end{aligned}
$$

Similarly, we have

$$
\begin{aligned}
\left\|\sum_{i} a_{i} z_{i}^{(1)}\right\|_{Z} & \leq C\left\|\sum_{i} a_{i} \cdot\right\| z_{i}^{(1)}\left\|_{Z} \cdot u_{\operatorname{minsupp}_{F}\left(z_{i}^{(1)}\right)}\right\|_{U} \\
& \leq B C\left\|\sum_{i} a_{i} \cdot\right\| z_{i}^{(1)}\left\|_{Z} \cdot u_{k_{i}}\right\|_{U}
\end{aligned}
$$

and

$$
\begin{aligned}
\left\|\sum_{i} a_{i} z_{i}^{(2)}\right\|_{V} & \leq B\left\|\sum_{i} a_{i} \cdot\right\| z_{i}^{(2)}\left\|_{Z} \cdot v_{k_{i}}\right\|_{V} \\
& \leq B D\left\|\sum_{i} a_{i} \cdot\right\| z_{i}^{(2)}\left\|_{Z} \cdot u_{k_{i}}\right\|_{U}
\end{aligned}
$$

It follows that

$$
\begin{aligned}
\left\|\sum_{i} a_{i} z_{i}\right\|_{W} & \leq \max \left\{B C\left\|\sum_{i} a_{i} \cdot\right\| z_{i}^{(1)}\left\|_{Z} \cdot u_{k_{i}}\right\|_{U}, B D\left\|\sum_{i} a_{i} \cdot\right\| z_{i}^{(2)}\left\|_{Z} \cdot u_{k_{i}}\right\|_{U}\right\} \\
& \leq B \cdot \max \{C, D\} \cdot\left\|\sum_{i} a_{i} u_{k_{i}}\right\|_{U} .
\end{aligned}
$$

The next two results show how norm estimates in a space and in its dual are related. 
Proposition 3. Assume that $Z$ has an FDD $\left(E_{i}\right)$, and let $V$ be a space with a normalized and 1-unconditional basis $\left(v_{i}\right)$. The following statements are equivalent:

(a) $\left(E_{i}\right)$ satisfies subsequential $V$-lower estimates in $Z$.

(b) $\left(E_{i}^{*}\right)$ satisfies subsequential $V^{(*)}$-upper estimates in $Z^{(*)}$.

(Here subsequential $V^{(*)}$-upper estimates are with respect to $\left(v_{i}^{*}\right)$, the sequence of biorthogonal functionals to $\left.\left(v_{i}\right)\right)$.

Moreover, if $\left(E_{i}\right)$ is bimonotone in $Z$, then the equivalence holds true if one replaces, for some $C \geq 1, V$-lower estimates by $C$-V-lower estimates in (a) and $V^{(*)}$-upper estimates by $C$ - $V^{(*)}$-upper estimates in (b).

Remark. By duality, Proposition 3 holds if we interchange the words lower and upper in (a) and (b).

Proof. Without loss of generality we may assume that $\left(E_{i}\right)$ is bimonotone in $Z$. "(a) $\Rightarrow(\mathrm{b})$ " Let $\left(z_{i}^{*}\right)$ be a normalized block sequence of $E^{*}=\left(E_{n}^{*}\right)$ in $Z^{(*)}$, and for each $i \in \mathbb{N}$ let $m_{i}=\min \operatorname{supp}_{E^{*}}\left(z_{i}^{*}\right)$. Given $\left(a_{i}\right) \in \mathrm{c}_{00}$, choose $z \in S_{Z}$ with finite support with respect to $\left(E_{n}\right)$ such that $\left\|\sum a_{i} z_{i}^{*}\right\|=\sum a_{i} z_{i}^{*}(z)$. For each $i \in \mathbb{N}$ write

$$
P_{\left[\min \operatorname{supp}_{E^{*}}\left(z_{i}^{*}\right), \min \operatorname{supp}_{E^{*}}\left(z_{i+1}^{*}\right)\right)}^{E}(z)=b_{i} z_{i},
$$

where $z_{i} \in S_{Z}$ and $\left|b_{i}\right| \leq 1$. Since $\left(E_{n}\right)$ satisfies subsequential $C$ - $V$-lower estimates in $Z$, we have $\left\|\sum b_{i} v_{m_{i}}\right\| \leq C\left\|\sum b_{i} z_{i}\right\| \leq C$. Hence

$$
\begin{aligned}
\left\|\sum a_{i} z_{i}^{*}\right\| & =\sum a_{i} b_{i} z_{i}^{*}\left(z_{i}\right) \leq \sum\left|a_{i} \| b_{i}\right| \\
& \leq\left\|\sum a_{i} v_{m_{i}}^{*}\right\| \cdot\left\|\sum b_{i} v_{m_{i}}\right\| \leq C\left\|\sum a_{i} v_{m_{i}}^{*}\right\|,
\end{aligned}
$$

as required.

"(b) $\Rightarrow(\mathrm{a})$ " Let $\left(z_{i}\right)$ be a normalized block sequence of $\left(E_{n}\right)$ in $Z$, and for each $i \in \mathbb{N}$ let $m_{i}=\min \operatorname{supp}_{E}\left(z_{i}\right)$. Given $\left(a_{i}\right) \in \mathrm{c}_{00}$, choose $\left(b_{i}\right) \in \mathrm{c}_{00}$ such that $\left\|\sum b_{i} v_{m_{i}}^{*}\right\|=$ 1 and $\left\|\sum a_{i} v_{m_{i}}\right\|=\sum a_{i} b_{i}$. For each $i \in \mathbb{N}$ there exists $z_{i}^{*} \in S_{Z^{(*)}}$ such that $z_{i}^{*}\left(z_{i}\right)=1$ and $\operatorname{ran}_{E^{*}}\left(z_{i}^{*}\right) \subset \operatorname{ran}_{E}\left(z_{i}\right)$. Since $\left(E_{n}^{*}\right)$ satisfies subsequential $C-V^{(*)}$ upper estimates in $Z^{(*)}$, we have $\left\|\sum b_{i} z_{i}^{*}\right\| \leq C$, and hence

$$
\left\|\sum a_{i} z_{i}\right\| \geq \frac{1}{C} \sum a_{i} b_{i}=\frac{1}{C}\left\|\sum a_{i} v_{m_{i}}\right\| .
$$

This completes the proof.

Proposition 4. Assume that $U$ is a space with a normalized, 1-unconditional basis $\left(u_{i}\right)$ which is $D$-right-dominant for some $D \geq 1$, and that $X$ is a reflexive space which satisfies subsequential $C$-U-upper tree estimates for some $C \geq 1$.

Then, for any $\varepsilon>0, X^{*}$ satisfies subsequential $(2 C D+\varepsilon)-U^{(*)}$-lower tree estimates.

Remark. One might ask whether or not the converse of Proposition 4 is true, i.e., similar to the FDD case, whether $X$ satisfies subsequential $U$-upper tree estimates if $X^{*}$ satisfies subsequential $U^{(*)}$-lower tree estimates.

The answer is affirmative under certain conditions on $U$, but we do not give a direct proof for that fact. Instead, we shall deduce it from one of our main embedding theorems (see Corollary 14 in Section 4).

Proof. We start with a simple observation. Let $\left(x_{i}^{*}\right)$ be a normalized, weakly null sequence in $X^{*}$. For each $n \in \mathbb{N}$ pick $x_{n} \in S_{X}$ with $x_{n}^{*}\left(x_{n}\right)=1$. There exist $y \in X$ 
and $k_{1}<k_{2}<\ldots$ in $\mathbb{N}$ such that $x_{k_{n}} \stackrel{w}{\rightarrow} y$. Given $\eta \in(0,1)$, there exists $n_{0} \in \mathbb{N}$ such that $\left|x_{k_{n}}^{*}(y)\right|<\eta$ for all $n \geq n_{0}$. Set

$$
y_{n}^{*}=x_{k_{n_{0}+n}}^{*} \quad \text { and } \quad y_{n}=\frac{x_{k_{n_{0}+n}}-y}{\left\|x_{k_{n_{0}+n}}-y\right\|}, \quad n \in \mathbb{N} .
$$

We have found, for given $\eta \in(0,1)$, a subsequence $\left(y_{i}^{*}\right)$ of $\left(x_{i}^{*}\right)$ and a normalized, weakly null sequence $\left(y_{i}\right)$ in $X$ satisfying $y_{n}^{*}\left(y_{n}\right)>(1-\eta) / 2$ for all $n \in \mathbb{N}$.

Now let $\left(x_{\alpha}^{*}\right)_{\alpha \in T_{\infty}^{\text {even }}}$ be a normalized, weakly null even tree in $X^{*}$. By the above observation we can find a normalized, weakly null even tree $\left(y_{\alpha}\right)_{\alpha \in T_{\infty}^{\text {even }}}$ in $X$ and a

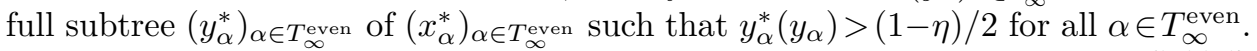
By a further pruning of these trees, we can also assume that $\left|y_{\alpha}^{*}\left(y_{\beta}\right)\right|<\eta / 2^{\max \{|\alpha|,|\beta|\}}$ whenever $\alpha<\beta$ or $\beta<\alpha$.

By assumption, there exist $m_{1}<m_{2}<\ldots$ in $\mathbb{N}$ such that $\left(y_{\left(m_{1}, m_{2}, \ldots, m_{2 i}\right)}\right)$ is $C$-dominated by $\left(u_{m_{2 i-1}}\right)$. Given $\left(a_{i}\right) \in \mathrm{c}_{00}$, there exists $\left(b_{i}\right) \in \mathrm{c}_{00}$ such that $\left\|\sum b_{i} u_{m_{2 i-1}}\right\|=1$ and $\sum a_{i} b_{i}=\left\|\sum a_{i} u_{m_{2 i-1}}^{*}\right\|$. So $\left\|\sum b_{i} y_{\left(m_{1}, m_{2}, \ldots, m_{2 i}\right)}\right\| \leq C$, and hence

$$
\begin{aligned}
\left\|\sum a_{i} y_{\left(m_{1}, m_{2}, \ldots, m_{2 i}\right)}^{*}\right\| & \geq \frac{1}{C}\left(\sum a_{i} b_{i} \frac{1-\eta}{2}-\sum_{i \neq j}\left|a_{i}\right|\left|b_{j}\right| \eta / 2^{\max \{i, j\}}\right) \\
& >\frac{1}{2 C+\varepsilon / D}\left\|\sum a_{i} u_{m_{2 i-1}}^{*}\right\|
\end{aligned}
$$

provided $\eta$ is sufficiently small. Now the branch $\left(y_{\left(m_{1}, m_{2}, \ldots, m_{2 i}\right)}^{*}\right)$ of $\left(y_{\alpha}^{*}\right)_{\alpha \in T_{\infty}^{\text {even }}}$ corresponds to a branch $\left(x_{\left(n_{1}, n_{2}, \ldots, n_{2 i}\right)}^{*}\right)$ of $\left(x_{\alpha}^{*}\right)_{\alpha \in T_{\infty}^{\text {even }}}$, where $n_{1}<n_{2}<\ldots$ and $m_{i} \leq n_{i}$ for all $i \in \mathbb{N}$. Since $\left(u_{i}\right)$ is $D$-right-dominant, it follows that $\left(u_{i}^{*}\right)$ is $D$-leftdominant, and hence the above inequality shows that $\left(x_{\left(n_{1}, n_{2}, \ldots, n_{2 i}\right)}^{*}(2 C D+\varepsilon)\right.$ dominates $\left(u_{n_{2 i-1}}^{*}\right)$.

We conclude this section with a key combinatorial result. We need to fix some terminology first.

Given a Banach space $X$, we let $\left(\mathbb{N} \times S_{X}\right)^{\omega}$ denote the set of all sequences $\left(k_{i}, x_{i}\right)$, where $k_{1}<k_{2}<\ldots$ are positive integers, and $\left(x_{i}\right)$ is a sequence in $S_{X}$. We equip the set $\left(\mathbb{N} \times S_{X}\right)^{\omega}$ with the product topology of the discrete topologies of $\mathbb{N}$ and $S_{X}$. Given $\mathcal{A} \subset\left(\mathbb{N} \times S_{X}\right)^{\omega}$ and $\varepsilon>0$, we let

$$
\mathcal{A}_{\varepsilon}=\left\{\left(\ell_{i}, y_{i}\right) \in\left(\mathbb{N} \times S_{X}\right)^{\omega}: \exists\left(k_{i}, x_{i}\right) \in \mathcal{A} \quad k_{i} \leq \ell_{i},\left\|x_{i}-y_{i}\right\|<\varepsilon \cdot 2^{-i} \forall i \in \mathbb{N}\right\},
$$

and we let $\overline{\mathcal{A}}$ be the closure of $\mathcal{A}$ in $\left(\mathbb{N} \times S_{X}\right)^{\omega}$.

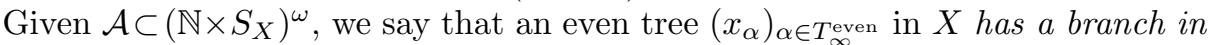
$\mathcal{A}$ if there exist $n_{1}<n_{2}<\ldots$ in $\mathbb{N}$ such that $\left(n_{2 i-1}, x_{\left(n_{1}, n_{2}, \ldots, n_{2 i}\right)}\right) \in \mathcal{A}$.

Proposition 5. Let $X$ be an infinite-dimensional (closed) subspace of a reflexive space $Z$ with an $F D D\left(E_{i}\right)$. Let $\mathcal{A} \subset\left(\mathbb{N} \times S_{X}\right)^{\omega}$. Then the following are equivalent.

(a) For all $\varepsilon>0$ every normalized, weakly null even tree in $X$ has a branch in $\overline{\mathcal{A}_{\varepsilon}}$.

(b) For all $\varepsilon>0$ there exist $\left(K_{i}\right) \subset \mathbb{N}$ with $K_{1}<K_{2}<\ldots, \bar{\delta}=\left(\delta_{i}\right) \subset(0,1)$ with $\delta_{i} \downarrow 0$, and a blocking $F=\left(F_{i}\right)$ of $\left(E_{i}\right)$ such that if $\left(x_{i}\right) \subset S_{X}$ is a $\bar{\delta}$-skipped block sequence of $\left(F_{n}\right)$ in $Z$ with $\left\|x_{i}-P_{\left(r_{i-1}, r_{i}\right)}^{F} x_{i}\right\|<\delta_{i}$ for all $i \in \mathbb{N}$, where $1 \leq r_{0}<r_{1}<r_{2}<\ldots$, then $\left(K_{r_{i-1}}, x_{i}\right) \in \overline{\mathcal{A}_{\varepsilon}}$.

Proof. For each $m \in \mathbb{N}$ we set $Z_{m}=\overline{\bigoplus_{i>m} E_{i}}$. Given $\varepsilon>0$, we consider the following game between players $\mathrm{S}$ (subspace chooser) and $\mathrm{P}$ (point chooser). The game has an infinite sequence of moves; on the $n^{\text {th }}$ move $(n \in \mathbb{N})$ S picks $k_{n}, m_{n} \in \mathbb{N}$ and $\mathrm{P}$ 
responds by picking $x_{n} \in S_{X}$ with $d\left(x_{n}, Z_{m_{n}}\right)<\varepsilon^{\prime} \cdot 2^{-n}$, where $\varepsilon^{\prime}=\min \{\varepsilon, 1\}$. S wins the game if the sequence $\left(k_{i}, x_{i}\right)$ the players generate ends up in $\overline{\mathcal{A}_{5 \varepsilon}}$, otherwise $\mathrm{P}$ is declared the winner. We will refer to this as the $(\mathcal{A}, \varepsilon)$-game and show that statements (a) and (b) above are equivalent to

(c) For all $\varepsilon>0 S$ has a winning strategy for the $(\mathcal{A}, \varepsilon)$-game.

Note that statement (b) yields a particular winning strategy for S, so the implication $(b) \Rightarrow(c)$ is clear, however this is included in the sequence of implications $(\mathrm{a}) \Rightarrow(\mathrm{c}) \Rightarrow(\mathrm{b}) \Rightarrow(\mathrm{a})$ which is what we are about to demonstrate.

"(a) $\Rightarrow(\mathrm{c})$ " Assume that for some $\varepsilon>0 \mathrm{~S}$ does not have a winning strategy for the $(\mathcal{A}, \varepsilon)$-game. Then there is a winning strategy $\phi$ for the point chooser $\mathrm{P}$. Thus $\phi$ is a function taking values in $S_{X}$ such that for all sequences $\left(k_{i}\right),\left(m_{i}\right)$ in $\mathbb{N}$ if $x_{n}=\phi\left(k_{1}, m_{1}, k_{2}, m_{2}, \ldots, k_{n}, m_{n}\right)$ for all $n \in \mathbb{N}$, then $d\left(x_{i}, Z_{m_{i}}\right)<\varepsilon^{\prime} \cdot 2^{-i}$ for all $i \in \mathbb{N}$ and $\left(k_{i}, x_{i}\right) \notin \overline{\mathcal{A}_{5 \varepsilon}}$. We will now construct a normalized, weakly null even tree $\left(x_{\alpha}\right)_{\alpha \in T_{\infty}^{\text {even }}}$ in $X$ to show that (a) fails. This will be a recursive construction which also builds auxiliary trees $\left(y_{\alpha}\right)_{\alpha \in T_{\infty}^{\text {even }}}$ in $X$ and $\left(m_{\alpha}\right)_{\alpha \in T_{\infty}^{\text {even }}}$ in $\mathbb{N}$.

Fix positive integers $\ell, n_{1}<n_{2}<\ldots<n_{2 \ell-1}$. Let $\alpha=\left(n_{1}, n_{2}, \ldots, n_{2 \ell-1}\right) \in T_{\infty}$, and for $1 \leq j \leq \ell$ set $k_{j}=n_{2 j-1}$. Assume that for $1 \leq j<\ell$ we have already defined $x_{j}=x_{\left(n_{1}, n_{2}, \ldots, n_{2 j}\right)}, y_{j}=y_{\left(n_{1}, n_{2}, \ldots, n_{2 j}\right)}$ and $m_{j}=m_{\left(n_{1}, n_{2}, \ldots, n_{2 j}\right)}$ such that $y_{j}=$ $\phi\left(k_{1}, m_{1}, k_{2}, m_{2}, \ldots, k_{j}, m_{j}\right)$. We will now construct the nodes $\left(x_{(\alpha, n)}\right),\left(y_{(\alpha, n)}\right)$ and $\left(m_{(\alpha, n)}\right)$. Set

$$
z_{i}=\phi\left(k_{1}, m_{1}, \ldots, k_{\ell-1}, m_{\ell-1}, k_{\ell}, i\right), \quad i \in \mathbb{N} .
$$

Note that $z_{i} \in S_{X}$ and $d\left(z_{i}, Z_{i}\right)<\varepsilon^{\prime} \cdot 2^{-\ell}$ for all $i \in \mathbb{N}$. We now pass to a weakly convergent subsequence: there exist $i_{1}<i_{2}<\ldots$ in $\mathbb{N}$ and $z \in X$ such that $z_{i_{n}} \stackrel{w}{\rightarrow} z$ as $n \rightarrow \infty$. Note that $\|z\| \leq \varepsilon^{\prime} \cdot 2^{-\ell}$. For each $n \in \mathbb{N}$ set

$$
w_{n}=\frac{z_{i_{n}}-z}{\left\|z_{i_{n}}-z\right\|} \text {. }
$$

Note that $\left(w_{n}\right)$ is a normalized, weakly null sequence in $X$, and

$$
\left\|z_{i_{n}}-w_{n}\right\| \leq \frac{2 \varepsilon^{\prime} \cdot 2^{-\ell}}{1-\varepsilon^{\prime} \cdot 2^{-\ell}} \leq 4 \varepsilon \cdot 2^{-\ell}
$$

for all $n \in \mathbb{N}$. We now set $x_{\left(n_{1}, n_{2}, \ldots, n_{2 \ell-1}, n\right)}=w_{n}, y_{\left(n_{1}, n_{2}, \ldots, n_{2 \ell-1}, n\right)}=z_{i_{n}}$ and $m_{\left(n_{1}, n_{2}, \ldots, n_{2 \ell-1}, n\right)}=i_{n}$ for all $n \in \mathbb{N}$ with $n>n_{2 \ell-1}$. This completes the recursive construction.

It follows by induction that $\left(x_{\alpha}\right)_{\alpha \in T_{\infty}^{\text {even }}}$ is a normalized, weakly null even tree in $X$ and $\left(y_{\alpha}\right)_{\alpha \in T_{\infty}^{\text {even }}}$ is a normalized even tree in $X$ such that for all $\alpha \in T_{\infty}^{\text {even }}$ we have $\left\|x_{\alpha}-y_{\alpha}\right\| \leq 4 \varepsilon \cdot 2^{-|\alpha| / 2}$. Moreover, given a sequence $n_{1}<n_{2}<\ldots$ in $\mathbb{N}$, setting $k_{j}=n_{2 j-1}, m_{j}=m_{\left(n_{1}, n_{2}, \ldots, n_{2 j}\right)}$ and $y_{j}=y_{\left(n_{1}, n_{2}, \ldots, n_{2 j}\right)}$ for all $j \in \mathbb{N}$, we have

$$
y_{n}=\phi\left(k_{1}, m_{1}, k_{2}, m_{2}, \ldots, k_{n}, m_{n}\right) \quad \text { for all } n \in \mathbb{N} \text {. }
$$

Hence no branch of $\left(y_{\alpha}\right)_{\alpha \in T_{\infty}^{\text {even }}}$ is in $\overline{\mathcal{A}_{5 \varepsilon}}$, and no branch of $\left(x_{\alpha}\right)_{\alpha \in T_{\infty}^{\text {even }}}$ is in $\overline{\mathcal{A}_{\varepsilon}}$. "(c) $\Rightarrow(\mathrm{b})$ " Let $(\phi, \psi)$ be a winning strategy for $S$ in the $(\mathcal{A}, \varepsilon)$-game. Thus $\phi$ and $\psi$ are functions taking values in $\mathbb{N}$ such that for all sequences $\left(k_{i}\right),\left(m_{i}\right)$ in $\mathbb{N}$ and $\left(x_{i}\right)$ in $S_{X}$ if $d\left(x_{n}, Z_{m_{n}}\right)<\varepsilon^{\prime} \cdot 2^{-n}, k_{n}=\phi\left(x_{1}, x_{2}, \ldots, x_{n-1}\right)$ and $m_{n} \geq \psi\left(x_{1}, x_{2}, \ldots, x_{n-1}\right)$ for all $n \in \mathbb{N}$, then $\left(k_{i}, x_{i}\right) \in \overline{\mathcal{A}_{5 \varepsilon}}$. For each interval $I \subset \mathbb{N}$ and $\delta>0$ fix a finite set $S_{I, \delta} \subset S_{X}$ such that for all $x \in S_{I, \delta}$ we have $\left\|x-P_{I}^{E} x\right\|<\delta$ and for all $y \in S_{X}$ if $\left\|y-P_{I}^{E} y\right\|<\delta$, then there exists $x \in S_{I, \delta}$ such that $\|x-y\|<3 \delta$.

We now construct a blocking $\left(F_{i}\right)$ of $\left(E_{i}\right)$ by recursion. Let $m_{1}=\psi()$ and $F_{1}=\bigoplus_{i=1}^{m_{1}} E_{i}$. Choose any $m_{2}>m_{1}$ and set $F_{2}=\bigoplus_{i=m_{1}+1}^{m_{2}} E_{i}$. Assume that for some $n \in \mathbb{N}, n \geq 3$, we have already chosen $m_{1}<\ldots<m_{n-1}$ and we have set 
$F_{j}=\bigoplus_{i=m_{j-1}+1}^{m_{j}} E_{i}$ for $1 \leq j<n\left(m_{0}=0\right)$. We now choose $m_{n}>m_{n-1}$ such that if $\ell \in \mathbb{N}, 1 \leq r_{0}<r_{1}<\ldots<r_{\ell} \leq n$ and

$$
x_{j} \in S_{\left[m_{r_{j-1}}+1, m_{r_{j}-1}\right], \varepsilon^{\prime} \cdot 2^{-j}} \quad \text { for } 1 \leq j \leq \ell,
$$

then $m_{n} \geq \psi\left(x_{1}, x_{2}, \ldots, x_{\ell}\right)$. Finally, we set $F_{n}=\bigoplus_{i=m_{n-1}+1}^{m_{n}} E_{i}$. This completes the recursive construction.

For each $n \in \mathbb{N}$ let $\delta_{n}=\varepsilon^{\prime} \cdot 2^{-n}$, and let $K_{n}$ be chosen so that $K_{n} \geq \phi()$, and if $\ell \in \mathbb{N}, 1 \leq r_{0}<r_{1}<\ldots<r_{\ell} \leq n$ and $x_{j} \in S_{\left[m_{r_{j-1}}+1, m_{r_{j}-1}\right], \varepsilon^{\prime} \cdot 2^{-j}}$ for $1 \leq j \leq \ell$, then $K_{n} \geq \phi\left(x_{1}, \ldots, x_{\ell}\right)$. We can of course also ensure that the sequence $\left(K_{i}\right)$ is strictly increasing. Let $\bar{\delta}=\left(\delta_{i}\right)$. We will now verify that (b) holds. Let $\left(y_{i}\right)$ be a $\bar{\delta}$-skipped block sequence of $\left(F_{n}\right)$ : there exist $1 \leq r_{0}<r_{1}<r_{2}<\ldots$ such that

$$
\left\|y_{i}-P_{\left[r_{i-1}+1, r_{i}-1\right]}^{F} y_{i}\right\|<\varepsilon^{\prime} \cdot 2^{-i} \quad \text { for all } i \in \mathbb{N},
$$

that is to say,

$$
\left\|y_{i}-P_{\left[m_{r_{i-1}}+1, m_{r_{i}-1}\right]}^{E} y_{i}\right\|<\varepsilon^{\prime} \cdot 2^{-i} \quad \text { for all } i \in \mathbb{N} .
$$

For each $i \in \mathbb{N}$ there exists $x_{i} \in S_{\left[m_{r_{i-1}}+1, m_{r_{i}-1}\right], \varepsilon^{\prime} \cdot 2^{-i}}$ such that $\left\|x_{i}-y_{i}\right\|<3 \varepsilon^{\prime} \cdot 2^{-i}$. Set

$$
k_{i}=\phi\left(x_{1}, \ldots, x_{i-1}\right) \quad \text { for each } i \in \mathbb{N} .
$$

Consider the sequence $k_{1}, m_{r_{0}}, x_{1}, k_{2}, m_{r_{1}}, x_{2}, \ldots$ We have $x_{i} \in S_{X}$ and

$$
d\left(x_{i}, Z_{m_{r_{i-1}}}\right) \leq\left\|x_{i}-P_{\left[m_{r_{i-1}}+1, m_{r_{i}-1}\right]}^{E} x_{i}\right\|<\varepsilon^{\prime} \cdot 2^{-i}
$$

for all $i \in \mathbb{N}$. Moreover $m_{r_{0}} \geq m_{1} \geq \psi(), K_{r_{0}} \geq K_{1} \geq \phi()=k_{1}$, and given $\ell \in \mathbb{N}$, setting $n=r_{\ell}$, we have $1 \leq r_{0}<r_{1}<\ldots<r_{\ell} \leq n$ and $x_{i} \in S_{\left[m_{r_{i-1}}+1, m_{r_{i}-1}\right], \varepsilon^{\prime} \cdot 2^{-i}}$ for $1 \leq i \leq \ell$. It follows that $\left(n \geq 3\right.$ and) $m_{r_{\ell}}=m_{n} \geq \psi\left(x_{1}, \ldots, x_{\ell}\right)$ and $k_{\ell+1} \leq K_{n}=K_{r_{\ell}}$. So $\left(k_{i}, x_{i}\right) \in \overline{\mathcal{A}_{5 \varepsilon}}$, and hence $\left(K_{r_{i-1}}, y_{i}\right) \in \overline{\mathcal{A}_{8 \varepsilon}}$.

"(b) $\Rightarrow(\mathrm{a})$ " Given $\varepsilon>0$, let $\left(K_{i}\right), \bar{\delta}=\left(\delta_{i}\right)$ and $\left(F_{i}\right)$ be as in statement (b). First note that if $\left(x_{i}\right)$ is a normalized, weakly null sequence in $X$, then

$$
\forall \eta>0 \forall p \in \mathbb{N} \quad \exists n \in \mathbb{N} \exists q>p \quad \text { such that } \quad\left\|x_{n}-P_{(p, q)}^{F} x_{n}\right\|<\eta .
$$

Indeed, the sequence $\left(P_{[1, p]}^{F} x_{i}\right)$ is weakly null, and hence norm-null, so there exists $n \in \mathbb{N}$ such that $\left\|P_{[1, p]}^{F} x_{n}\right\|<\eta / 2$. One can then choose $q>p$ such that $\left\|P_{[q, \infty)}^{F} x_{n}\right\|<$ $\eta / 2$. The claim now follows by triangle-inequality.

Now let $\left(x_{\alpha}\right)_{\alpha \in T_{\infty}^{\text {even }}}$ be a normalized, weakly null even tree in $X$. We choose positive integers $n_{1}<n_{2}<\ldots$ and $1=r_{0}<r_{1}<r_{2}<\ldots$ by recursion. For $\ell \in \mathbb{N}$ we first choose $n_{2 \ell-1}>K_{r_{\ell-1}}$ such that $n_{2 \ell-1}>n_{2 \ell-2}\left(n_{0}=0\right)$, and then choose $n_{2 \ell}>n_{2 \ell-1}$ and $r_{\ell}>r_{\ell-1}$ such that

$$
\left\|x_{\left(n_{1}, n_{2}, \ldots, n_{2 \ell}\right)}-P_{\left(r_{\ell-1}, r_{\ell}\right)}^{F} x_{\left(n_{1}, n_{2}, \ldots, n_{2 \ell}\right)}\right\|<\delta_{\ell} .
$$

By assumption (b) we have $\left(n_{2 i-1}, x_{\left(n_{1}, n_{2}, \ldots, n_{2 i}\right)}\right) \in \overline{\mathcal{A}_{\varepsilon}}$.

\section{THE SPACE $Z^{V}(E)$}

Let $Z$ be a space with an FDD $E=\left(E_{i}\right)$, and let $V$ be a space with a 1 unconditional and normalized basis $\left(v_{i}\right)$. The space $Z^{V}=Z^{V}(E)$ is defined to be the completion of $\mathrm{c}_{00}\left(\oplus E_{i}\right)$ with respect to the following norm $\|\cdot\|_{Z^{v}}$.

$$
\|z\|_{Z^{V}}=\max _{\substack{k \in \mathbb{N} \\ 1 \leq n_{0}<n_{1}<n_{2}<\cdots<n_{k}}}\left\|\sum_{j=1}^{k}\right\| P_{\left[n_{j-1}, n_{j}\right)}^{E}(z)\left\|_{Z} \cdot v_{n_{j-1}}\right\|_{V} \quad \text { for } z \in \mathrm{c}_{00}\left(\oplus E_{i}\right) .
$$

Note that if $\left(v_{i}\right)$ is $C$-block-stable and $D$-right-dominant, then the projection constant $K\left(E, Z^{V}\right)$ of $\left(E_{i}\right)$ in $Z^{V}$ satisfies

$$
K\left(E, Z^{V}\right) \leq \min \{K(E, Z), C, D, 2\} .
$$


Here we allow $C=\infty$ or $D=\infty$ if $\left(v_{i}\right)$ is not block-stable or not right-dominant, respectively. Note also that if $\|\cdot\|$ and $\|\cdot\|^{\prime}$ are equivalent norms on $Z$, then the corresponding norms $\|\cdot\|_{Z^{V}}$ and $\|\cdot\|_{Z^{V}}^{\prime}$ are equivalent on $c_{00}\left(\oplus E_{i}\right)$. This often allows us, when examining the space $Z^{V}$, to assume that $\left(E_{i}\right)$ is bimonotone in $Z$.

Our first set of results culminating in Corollary 9 determine when the space $Z^{V}(E)$ is reflexive.

Lemma 6. Every normalized block sequence $\left(z_{i}\right)$ of $\left(E_{n}\right)$ in $Z^{V} 1$-dominates some block sequence $\left(b_{i}\right)$ of $\left(v_{n}\right)$ that satisfies

$$
1 / 2 \leq\left\|b_{i}\right\|_{V} \leq 1 \quad \text { and } \quad \operatorname{ran}\left(b_{i}\right) \subset \operatorname{ran}_{E}\left(z_{i}\right) \quad \text { for all } i \in \mathbb{N} .
$$

(Here the range, $\operatorname{ran}(x)$, of $x=\sum a_{i} v_{i} \in V$ is the smallest interval in $\mathbb{N}$ containing $\left\{i \in \mathbb{N}: a_{i} \neq 0\right\}$.)

Proof. Let $z \in S_{Z^{V}}$ have finite support with respect to $\left(E_{i}\right)$. Choose $k, 1 \leq n_{0}<$ $n_{1}<\ldots<n_{k}$ in $\mathbb{N}$ such that

$$
\|z\|_{Z^{V}}=\left\|\sum_{j=1}^{k}\right\| P_{\left[n_{j-1}, n_{j}\right)}^{E}(z)\left\|_{Z} \cdot v_{n_{j-1}}\right\|_{V} .
$$

Without loss of generality we can assume that $n_{0} \leq \min \operatorname{supp}_{E}(z) \leq n_{1}-1$ and that $n_{k-1} \leq \max \operatorname{supp}_{E}(z)=n_{k}-1$. Set $m_{0}=\min \operatorname{supp}_{E}(z), m_{j}=n_{j}$ for $1 \leq j<k$, and let $m_{k}>\max \operatorname{supp}_{E}(z)$. By the triangle-inequality we have

$$
\begin{aligned}
\|z\|_{Z^{V}} & \leq\|\| P_{\left[n_{0}, n_{1}\right)}^{E}(z)\left\|_{Z} \cdot v_{n_{0}}\right\|_{V}+\left\|\sum_{j=2}^{k}\right\| P_{\left[n_{j-1}, n_{j}\right)}^{E}(z)\left\|_{Z} \cdot v_{n_{j-1}}\right\|_{V} \\
& \leq 2\left\|\sum_{j=1}^{k}\right\| P_{\left[m_{j-1}, m_{j}\right)}^{E}(z)\left\|_{Z} \cdot v_{m_{j-1}}\right\|_{V} .
\end{aligned}
$$

Now let $\left(z_{i}\right)$ be a normalized block sequence of $\left(E_{n}\right)$ in $Z^{V}$. It follows from the above that there exist positive integers $1 \leq n_{0}<n_{1}<\ldots$ and $1=k_{1}<k_{2}<\ldots$ such that $n_{k_{\ell}-1}=\min \operatorname{supp}_{E}\left(z_{\ell}\right), n_{k_{\ell+1}-2} \leq \max \operatorname{supp}_{E}\left(z_{\ell}\right)$, and

$$
\left\|z_{\ell}\right\|_{Z^{V}} \leq 2\left\|\sum_{j=k_{\ell}}^{k_{\ell+1}-1}\right\| P_{\left[n_{j-1}, n_{j}\right)}^{E}\left(z_{\ell}\right)\left\|_{Z} \cdot v_{n_{j-1}}\right\|_{V} \quad \text { for all } \ell \in \mathbb{N} .
$$

It follows that

$$
b_{\ell}=\sum_{j=k_{\ell}}^{k_{\ell+1}-1}\left\|P_{\left[n_{j-1}, n_{j}\right)}^{E}\left(z_{\ell}\right)\right\|_{Z} \cdot v_{n_{j-1}}
$$

satisfies $1 / 2 \leq\left\|b_{\ell}\right\|_{V} \leq 1$ and $\operatorname{ran}\left(b_{\ell}\right) \subset \operatorname{ran}_{E}\left(z_{\ell}\right)$ for all $\ell \in \mathbb{N}$. Moreover, given $\left(a_{i}\right) \in \mathrm{c}_{00}$, setting $z=\sum a_{i} z_{i}$ we have

$$
\begin{aligned}
\|z\|_{Z^{V}} & \geq\left\|\sum_{j=1}^{\infty}\right\| P_{\left[n_{j-1}, n_{j}\right)}^{E}(z)\left\|_{Z} \cdot v_{n_{j-1}}\right\|_{V} \\
& =\left\|\sum_{\ell=1}^{\infty} \sum_{j=k_{\ell}}^{k_{\ell+1}-1}\right\| P_{\left[n_{j-1}, n_{j}\right)}^{E}(z)\left\|_{Z} \cdot v_{n_{j-1}}\right\|_{V} \\
& =\left\|\sum_{\ell=1}^{\infty} a_{\ell} b_{\ell}\right\|_{V} .
\end{aligned}
$$


Corollary 7. Let $V$ be a Banach space with a normalized and 1-unconditional basis $\left(v_{i}\right)$, and let $Z$ be a space with an FDD $E=\left(E_{i}\right)$.

If the basis $\left(v_{i}\right)$ is boundedly complete, then $\left(E_{i}\right)$ is a boundedly complete FDD for $Z^{V}(E)$.

Proof. Let $\left(z_{i}\right)$ be a normalized block sequence of $\left(E_{n}\right)$ in $Z^{V}$. Let $\left(b_{i}\right)$ be a block sequence of $\left(v_{n}\right)$ given by Lemma 6. Given $\varepsilon>0$, let $\left(a_{i}\right)$ be a scalar sequence with $\left|a_{i}\right|>\varepsilon$ for all $i \in \mathbb{N}$. Since $\left(v_{i}\right)$ is boundedly complete, and since $\left(z_{i}\right)$ dominates $\left(b_{i}\right)$ it follows that

$$
\sup _{n}\left\|\sum_{i=1}^{n} a_{i} z_{i}\right\|_{Z^{V}}=\infty .
$$

Hence $\left(E_{i}\right)$ is a boundedly complete FDD for $Z^{V}(E)$.

Lemma 8. Let $V$ be a Banach space with a normalized and 1-unconditional basis $\left(v_{i}\right)$, and assume that the space $Z$ has an FDD $E=\left(E_{i}\right)$.

If the basis $\left(v_{i}\right)$ is shrinking and if $\left(E_{i}\right)$ is a shrinking FDD for $Z$ then $\left(E_{i}\right)$ is also a shrinking FDD for $Z^{V}(E)$.

Proof. Without loss of generality we may assume that $\left(E_{i}\right)$ is bimonotone in $Z$. We first note that, given positive integers $1 \leq n_{0}<n_{1}<\ldots$ and vectors $z_{j}^{*} \in$ $\bigoplus_{i \in\left[n_{j-1}, n_{j}\right)} E_{i}^{*}$ with $\left\|z_{j}^{*}\right\|_{Z^{*}} \leq 1$ for each $j \in \mathbb{N}$, if $v^{*}=\sum_{i=1}^{\infty} a_{i} v_{n_{i-1}}^{*}$ converges in $V^{*}$ with $\left\|v^{*}\right\| \leq 1$, then the series $z^{*}=\sum_{i=1}^{\infty} a_{i} z_{i}^{*}$ converges in $\left(Z^{V}\right)^{*}$ and $\left\|z^{*}\right\|_{\left(Z^{V}\right)^{*}} \leq 1$. Indeed, for $p \leq q$ in $\mathbb{N}$ there exists $z \in S_{Z^{V}}$ with $\operatorname{supp}_{E}(z) \subset\left[n_{p-1}, n_{q}\right)$ such that

$$
\begin{aligned}
\left\|\sum_{j=p}^{q} a_{j} z_{j}^{*}\right\|_{\left(Z^{V}\right)^{*}} & =\sum_{j=p}^{q} a_{j} z_{j}^{*}(z) \leq \sum_{j=p}^{q}\left|a_{j}\right| \cdot\left\|P_{\left[n_{j-1}, n_{j}\right)}^{E}(z)\right\|_{Z} \\
& \leq\left\|\sum_{j=p}^{q} a_{j} v_{n_{j-1}}^{*}\right\|_{V^{*}} \cdot\left\|\sum_{j=p}^{q}\right\| P_{\left[n_{j-1}, n_{j}\right)}^{E}(z)\left\|_{Z} \cdot v_{n_{j-1}}\right\|_{V} \\
& \leq\left\|\sum_{j=p}^{q} a_{j} v_{n_{j-1}}^{*}\right\|_{V^{*}} \cdot\|z\|_{Z^{V}} \leq\left\|\sum_{j=p}^{q} a_{j} v_{n_{j-1}}^{*}\right\|_{V^{*}},
\end{aligned}
$$

which implies the claim. Next define $K$ to be the union of the following two sets $K_{1}$ and $K_{2}$ :

$$
\begin{gathered}
K_{1}=\left\{\sum_{i=1}^{\infty} a_{i} z_{i}^{*}: 1 \leq n_{0}<n_{1}<\ldots, \quad z_{j}^{*} \in \bigoplus_{i \in\left[n_{j-1}, n_{j}\right)} E_{i}^{*} \text { and }\left\|z_{j}^{*}\right\|_{Z^{*}} \leq 1 \forall j \in \mathbb{N},\right. \\
K_{2}=\left\{\sum_{i=1}^{\infty} a_{i} v_{n_{i-1}}^{*} \|_{V^{*}} \leq 1\right\}, \\
\left.\| \sum_{i=1}^{\ell} a_{i} z_{i}^{*}: \ell \in \mathbb{N}, 1 \leq n_{0}<n_{1}<\ldots<n_{\ell} \leq \infty, \quad z_{j}^{*} \in \bigoplus_{i \in\left[n_{j-1}, n_{j}\right)} E_{i}^{*} \text { and } \text { for } 1 \leq j \leq \ell,\left\|\sum_{i=1}^{\ell} a_{i} v_{n_{i-1}}^{*}\right\|_{V^{*}} \leq 1\right\} .
\end{gathered}
$$

An element $\sum_{i=1}^{\ell} a_{i} z_{i}^{*}$ of $K_{2}$ will also be written as an infinite sum $\sum_{i=1}^{\infty} a_{i} z_{i}^{*}$ by setting $a_{i}=0$ and $z_{i}^{*}=0$ for all $i>\ell$. Clearly, $K$ is a $Z^{V}$-norming subset (isometrically) of $B_{\left(Z^{V}\right)^{*}}$. We claim that $K$ is $w^{*}$-compact. Indeed, for each $k \in \mathbb{N}$ let $y_{k}^{*}=\sum_{i=1}^{\infty} a_{i}^{(k)} z_{(k, i)}^{*} \in K$, where for some (finite or infinite) sequence $1 \leq n_{0}^{(k)}<$ $n_{1}^{(k)}<n_{2}^{(k)}<\ldots$ in $\mathbb{N} \cup\{\infty\}$ we have $z_{(k, j)}^{*} \in \bigoplus_{i \in\left[n_{j-1}^{(k)}, n_{j}^{(k)}\right)} E_{i}^{*}$ and $\left\|z_{(k, j)}^{*}\right\|_{Z^{*}} \leq 1$ 
for all $j$, and $\left\|\sum_{i} a_{i}^{(k)} v_{n_{i-1}^{*}}^{*}\right\|_{V^{*}} \leq 1$. After passing to a subsequence we can assume that

$$
a_{i}=\lim _{k \rightarrow \infty} a_{i}^{(k)} \quad \text { exists for all } i \in \mathbb{N} \text {, and }
$$

there exists $\ell \in \mathbb{N} \cup\{0, \infty\}$ such that

$$
\begin{aligned}
\lim _{k \rightarrow \infty} n_{j}^{(k)} & =n_{j} & & \text { exists for } 0 \leq j<\ell, \\
\lim _{k \rightarrow \infty} n_{\ell}^{(k)} & =\infty & & \text { if } \ell \in \mathbb{N} \cup\{0\}, \\
\lim _{k \rightarrow \infty} z_{(k, j)}^{*} & =z_{j}^{*} & & \text { exists (in norm) for } 0 \leq j<\ell, \\
w^{*}-\lim _{k \rightarrow \infty} z_{(k, \ell)}^{*} & =z_{\ell}^{*} & & \text { exists if } \ell \in \mathbb{N} \cup\{0\}, \text { and } \\
w^{*}-\lim _{k \rightarrow \infty} \sum_{i \in \mathbb{N}, i>\ell} a_{i}^{(k)} z_{(k, i)}^{*} & =0 & & \text { if } \ell \in \mathbb{N} \cup\{0\} .
\end{aligned}
$$

Consider the case when $\ell=\infty$. We have $1 \leq n_{0}<n_{1}<\ldots, z_{j}^{*} \in \bigoplus_{\left[n_{j-1}, n_{j}\right)} E_{i}^{*}$ and $\left\|z_{j}^{*}\right\|_{Z^{*}} \leq 1$ for all $j \in \mathbb{N}$. Moreover, since $\left(v_{i}^{*}\right)$ is a boundedly complete basis of $V^{*}$, the series $\sum_{i=1}^{\infty} a_{i} v_{n_{i-1}}^{*}$ converges and $\left\|\sum_{i=1}^{\infty} a_{i} v_{n_{i-1}}^{*}\right\|_{V^{*}} \leq 1$. Hence $z^{*}=\sum_{i=1}^{\infty} a_{i} z_{i}^{*}$ belongs to $K$. Finally, given $z \in S_{Z}$ with finite support with respect to $\left(E_{i}\right)$, for sufficiently large $r \in \mathbb{N}$ we have

$$
\begin{aligned}
\lim _{k \rightarrow \infty} y_{k}^{*}(z) & =\lim _{k \rightarrow \infty} \sum_{i=1}^{r} a_{i}^{(k)} z_{(k, i)}^{*}(z) \\
& =\sum_{i=1}^{r} a_{i} z_{i}^{*}(z)=z^{*}(z) .
\end{aligned}
$$

It follows that $y_{k}^{*} \stackrel{w^{*}}{\rightarrow} z^{*}$ as $k \rightarrow \infty$. The case $\ell \in \mathbb{N} \cup\{0\}$ is similar. We have $1 \leq n_{0}<n_{1}<\ldots<n_{\ell} \leq \infty, z_{j}^{*} \in \bigoplus_{\left[n_{j-1}, n_{j}\right)} E_{i}^{*}$ and $\left\|z_{j}^{*}\right\|_{Z^{*}} \leq 1$ for $1 \leq j \leq \ell$, and $\sum_{i=1}^{\ell} a_{i} v_{n_{i-1}}^{*} \in B_{V^{*}}$. So $z^{*}=\sum_{i=1}^{\ell} a_{i} z_{i}^{*} \in K$ and $y_{k}^{*} \stackrel{w^{*}}{\rightarrow} z^{*}$ as $k \rightarrow \infty$. This completes the proof that $K$ is $w^{*}$-closed.

We deduce that $Z^{V}$ is embedded in $C(K)$, the space of continuous functions on $K$. Let $\left(z_{i}\right)$ be a bounded block sequence of $\left(E_{n}\right)$ in $Z^{V}$, and let $z^{*} \in K$. Using the notation as in the definition of $K$, if $z^{*} \in K_{1}$, then computing as in (1)

$$
z^{*}\left(z_{i}\right)=\sum_{j, n_{j} \geq \operatorname{minsupp}_{E}\left(z_{i}\right)} a_{j} z_{j}^{*}\left(z_{i}\right) \leq\left\|z_{i}\right\|_{Z^{V}} \cdot\left\|\sum_{j, n_{j} \geq \operatorname{minsupp}_{E}\left(z_{i}\right)} a_{j} v_{n_{j-1}}^{*}\right\|_{V^{*}},
$$

which converges to zero as $i \rightarrow \infty$; and if $z^{*} \in K_{2}$, then for all sufficiently large values of $i$

$$
z^{*}\left(z_{i}\right)=\sum_{j=1}^{\ell} a_{j} z_{j}^{*}\left(z_{i}\right)=a_{\ell} z_{\ell}^{*}\left(z_{i}\right)
$$

which converges to zero as $i \rightarrow \infty$, since $\left(E_{i}\right)$ is assumed a shrinking FDD for $Z$.

It follows that $\left(z_{i}\right)$ is weakly null in $C(K)$, and thus in $Z^{V}$. Since $\left(z_{i}\right)$ was an arbitrary bounded block sequence in $Z^{V}$, this finishes the proof that $\left(E_{i}\right)$ is shrinking in $Z^{V}$.

From Lemma 8 and Corollary 7 we obtain the following result.

Corollary 9. Assume that $V$ is a reflexive Banach space with a normalized and 1-unconditional basis $\left(v_{i}\right)$ and that $Z$ is a space with a shrinking $F D D E=\left(E_{i}\right)$. Then $Z^{V}(E)$ is reflexive.

The idea of the norm $\|\cdot\|_{Z^{V}}$ is, of course, to introduce a subsequential $V$-lowerestimate. The next lemma determines when this is the case. 
Lemma 10. Let $V$ be a Banach space with a normalized and 1-unconditional basis $\left(v_{i}\right)$, and let $Z$ be a Banach space with an FDD $E=\left(E_{i}\right)$.

If, for some $C \geq 1,\left(v_{i}\right)$ is $C$-block stable, then $\left(E_{i}\right)$ satisfies subsequential $2 C$ - $V$ lower estimates in $Z^{V}(E)$.

Proof. Let $\left(z_{i}\right)$ be a normalized block sequence in $Z^{V}(E)$, and for each $i \in \mathbb{N}$ let $m_{i}=\min \operatorname{supp}_{E}\left(z_{i}\right)$. By Lemma 6, there exists a block sequence $\left(b_{i}\right)$ of $\left(v_{n}\right)$ with $1 / 2 \leq\left\|b_{i}\right\|_{V} \leq 1$ and $\operatorname{ran}\left(b_{i}\right) \subset \operatorname{ran}_{E}\left(z_{i}\right)$ for all $i \in \mathbb{N}$, which is 1-dominated by $\left(z_{i}\right)$. Since $\left(v_{i}\right)$ is 1 -unconditional and $C$-block-stable, it follows that $\left(b_{i}\right) 2 C$-dominates $\left(v_{m_{i}}\right)$, which proves the lemma.

The final result in this section shows when subsequential $U$-upper estimates are preserved under $Z \mapsto Z^{V}$.

Lemma 11. Let $V$ and $U$ be Banach spaces with normalized, 1-unconditional and block-stable bases $\left(v_{i}\right)$ and $\left(u_{i}\right)$, respectively, and assume that $\left(v_{i}\right)$ is dominated by $\left(u_{i}\right)$. Let $Z$ be a Banach space with an FDD $\left(E_{i}\right)$.

If $\left(E_{i}\right)$ satisfies subsequential $U$-upper estimates in $Z$, then $\left(E_{i}\right)$ also satisfies subsequential $U$-upper estimates in $Z^{V}$.

Proof. Choose constants $B_{V}, B_{U}, D$ and $C$ in $[1, \infty)$ such that $\left(v_{i}\right)$ is $B_{V}$-blockstable, $\left(u_{i}\right)$ is $B_{U}$-block-stable, $\left(v_{i}\right)$ is $D$-dominated by $\left(u_{i}\right)$, and $\left(E_{i}\right)$ satisfies subsequential $C$ - $U$-upper estimates in $Z$. Let $K$ be the projection constant of $\left(E_{i}\right)$ in $Z$, and set $\bar{C}=B_{V} D+B_{U} C D+2 B_{V} D K$. We show that for any finite block sequence $\left(z_{i}\right)_{i=1}^{\ell}$ of $\left(E_{n}\right)$, and for any $k$ and $n_{0}<n_{1}<\ldots<n_{k}$ in $\mathbb{N}$ we have (putting $z=\sum_{i=1}^{\ell} z_{i}$ and $m_{j}=\min \operatorname{supp}_{E}\left(z_{j}\right)$ for $\left.1 \leq j \leq \ell\right)$

$$
\left\|\sum_{j=1}^{k}\right\| P_{\left[n_{j-1}, n_{j}\right)}^{E}(z)\left\|_{Z} \cdot v_{n_{j-1}}\right\|_{V} \leq \bar{C} \cdot\left\|\sum_{i=1}^{\ell}\right\| z_{i}\left\|_{Z^{V}} \cdot u_{m_{i}}\right\|_{U} .
$$

Taking then the supremum of the left side of (2) over all choices of $k$ and $n_{0}<n_{1}<$ $\ldots<n_{k}$ in $\mathbb{N}$, we obtain

$$
\left\|\sum_{i=1}^{\ell} z_{i}\right\|_{Z^{V}} \leq \bar{C} \cdot\left\|\sum_{i=1}^{\ell}\right\| z_{i}\left\|_{Z^{V}} \cdot u_{m_{i}}\right\|_{U}
$$

and thus that $\left(E_{i}\right)$ satisfies subsequential $\bar{C}$ - $U$-upper estimates in $Z^{V}$. Note that in proving (2) we can of course assume that $n_{k} \leq \max \operatorname{supp}_{E}\left(z_{\ell}\right)+1$.

For $i=1,2, \ldots, \ell$ put

$$
J_{i}=\left\{j \in\{1,2, \ldots, k\}: \min \operatorname{supp}_{E}\left(z_{i}\right) \leq n_{j-1}<n_{j} \leq \min \operatorname{supp}_{E}\left(z_{i+1}\right)\right\}
$$

$\left(\right.$ with $\left.\min \operatorname{supp}_{E}\left(z_{\ell+1}\right)=\max \operatorname{supp}_{E}\left(z_{\ell}\right)+1\right)$ and $J_{0}=\{1,2, \ldots, k\} \backslash \bigcup_{i=1}^{\ell} J_{i}$.

For $j=1,2, \ldots, k$ put

$$
I_{j}=\left\{i \in\{1,2, \ldots, \ell\}: n_{j-1} \leq \min \operatorname{supp}_{E}\left(z_{i}\right) \leq \max \operatorname{supp}_{E}\left(z_{i}\right)<n_{j}\right\}
$$

and $I_{0}=\{1,2, \ldots, \ell\} \backslash \bigcup_{j=1}^{k} I_{j}$.

Firstly, we have

$$
\left\|\sum_{i=1}^{\ell} \sum_{j \in J_{i}}\right\| P_{\left[n_{j-1}, n_{j}\right)}^{E}\left(z_{i}\right)\left\|_{Z} \cdot v_{n_{j-1}}\right\|_{V}=\left\|\sum_{i=1}^{\ell} b_{i}\right\|_{V}
$$


(where $b_{i}=\sum_{j \in J_{i}}\left\|P_{\left[n_{j-1}, n_{j}\right)}^{E}\left(z_{i}\right)\right\|_{Z} \cdot v_{n_{j-1}}$ for $1 \leq i \leq \ell$ )

$$
\begin{aligned}
& \leq B_{V}\left\|\sum_{i=1}^{\ell}\right\| b_{i}\left\|_{V} \cdot v_{m_{i}}\right\|_{V} \\
& \leq B_{V} D\left\|\sum_{i=1}^{\ell}\right\| z_{i}\left\|_{Z^{V}} \cdot u_{m_{i}}\right\|_{U} .
\end{aligned}
$$

Secondly,

(4) $\left\|\sum_{j \in J_{0}}\right\| \sum_{i \in I_{j}} z_{i}\left\|_{Z} \cdot v_{n_{j-1}}\right\|_{V} \leq C\left\|\sum_{j \in J_{0}}\right\| b_{j}\left\|_{U} \cdot v_{n_{j-1}}\right\|_{V}$

(where $b_{j}=\sum_{i \in I_{j}}\left\|z_{i}\right\|_{Z} \cdot u_{m_{i}}$ for each $j \in J_{0}$ )

$$
\begin{aligned}
& \leq C D\left\|\sum_{j \in J_{0}}\right\| b_{j}\left\|_{U} \cdot u_{n_{j-1}}\right\|_{U} \\
& \leq C D B_{U}\left\|\sum_{j \in J_{0}} b_{j}\right\|_{U} \leq C D B_{U}\left\|\sum_{i=1}^{\ell}\right\| z_{i}\left\|_{Z^{v}} \cdot u_{m_{i}}\right\|_{U} .
\end{aligned}
$$

Thirdly, given $j \in J_{0}$ and $i \in I_{0}$ such that $P_{\left[n_{j-1}, n_{j}\right)}^{E}\left(z_{i}\right) \neq 0$, we have either

$$
n_{j-1}<\min \operatorname{supp}_{E}\left(z_{i}\right)<n_{j} \leq \max \operatorname{supp}_{E}\left(z_{i}\right), \quad \text { or }
$$

$$
\min \operatorname{supp}_{E}\left(z_{i}\right)<n_{j-1} \leq \max \operatorname{supp}_{E}\left(z_{i}\right)<n_{j}
$$

Let $J_{0,1}$ be the set of all $j \in J_{0}$ for which there exists an $i \in I_{0}$ such that (5) holds and let $i_{j}^{1}$ denote the unique such $i \in I_{0}$. Similarly, we let $J_{0,2}$ be the set of all $j \in J_{0}$ for which there exists an $i \in I_{0}$ such that (6) holds and we denote by $i_{j}^{2}$ the unique such $i \in I_{0}$. We now obtain

$$
\begin{aligned}
\left\|\sum_{j \in J_{0}} \sum_{i \in I_{0}}\right\| P_{\left[n_{j-1}, n_{j}\right)}^{E} & \left(z_{i}\right)\left\|_{Z} \cdot v_{n_{j-1}}\right\|_{V} \\
& \leq K\left\|\sum_{j \in J_{0,1}}\right\| z_{i_{j}^{1}}\left\|{ }_{Z} \cdot v_{n_{j-1}}\right\|_{V}+K\left\|\sum_{j \in J_{0,2}}\right\| z_{i_{j}^{2}}\left\|Z \cdot v_{n_{j-1}}\right\|_{V} \\
& \leq K B_{V}\left\|\sum_{j \in J_{0,1}}\right\| z_{i_{j}^{1}}\left\|_{Z} \cdot v_{m_{i_{j}^{1}}}\right\|_{V}+K B_{V}\left\|\sum_{j \in J_{0,2}}\right\| z_{i_{j}^{2}}\left\|_{Z} \cdot v_{m_{i_{j}^{2}}}\right\|_{V} \\
& \leq 2 K B_{V} D\left\|\sum_{i=1}^{\ell}\right\| z_{i}\left\|_{Z^{V}} \cdot u_{m_{i}}\right\|_{U} .
\end{aligned}
$$


Finally, we deduce from (3), (4) and (7) that

$$
\begin{aligned}
\left\|\sum_{j=1}^{k}\right\| P_{\left[n_{j-1}, n_{j}\right)}^{E}(z)\left\|_{Z} \cdot v_{n_{j-1}}\right\|_{V} \\
\leq \| \\
\quad \sum_{i=1}^{\ell} \sum_{j \in J_{i}}\left\|P_{\left[n_{j-1}, n_{j}\right)}^{E}\left(z_{i}\right)\right\|_{Z} \cdot v_{n_{j-1}} \|_{V} \\
\quad+\left\|\sum_{j \in J_{0}}\right\| P_{\left[n_{j-1}, n_{j}\right)}^{E}(z)\left\|_{Z} \cdot v_{n_{j-1}}\right\|_{V} \\
\leq\left\|\sum_{i=1}^{\ell} \sum_{j \in J_{i}}\right\| P_{\left[n_{j-1}, n_{j}\right)}^{E}\left(z_{i}\right)\left\|_{Z} \cdot v_{n_{j-1}}\right\|_{V} \\
\quad+\left\|\sum_{j \in J_{0}}\right\| \sum_{i \in I_{j}} z_{i}\left\|_{Z} \cdot v_{n_{j-1}}\right\|_{V} \\
\quad+\left\|\sum_{j \in J_{0}} \sum_{i \in I_{0}}\right\| P_{\left[n_{j-1}, n_{j}\right)}^{E}\left(z_{i}\right)\left\|_{Z} \cdot v_{n_{j-1}}\right\|_{V} \\
\leq\left(B_{V} D+B_{U} C D+2 B_{V} D K\right)\left\|\sum_{i=1}^{m}\right\| z_{i}\left\|_{Z^{V}} \cdot u_{m_{i}}\right\|_{U}
\end{aligned}
$$

which finishes the proof of (2).

\section{EMBEDDING THEOREMS}

In this section we will prove and deduce some consequences of

Theorem 12. Assume that $V$ is a Banach space with a normalized, 1-unconditional and left-dominant basis $\left(v_{i}\right)$. Let $X$ be a separable, infinite-dimensional, reflexive space with subsequential $V$-lower tree estimates.

(a) For every reflexive space $Z$ with an FDD $E=\left(E_{i}\right)$ which contains $X$ there is a blocking $H=\left(H_{i}\right)$ of $\left(E_{i}\right)$, and there exists $N \in \mathbb{N}^{(\omega)}$ such that $X$ naturally isomorphically embeds into $Z^{V_{N}}(H)$.

(b) There is a space $\tilde{Y}$ with a bimonotone, shrinking $F D D \tilde{G}=\left(\tilde{G}_{i}\right)$, and there exists $N \in \mathbb{N}^{(\omega)}$ such that $X$ is a quotient of $\tilde{Y}^{V_{N}}(\tilde{G})$.

Recall that $\mathbb{N}^{(\omega)}$ denotes the set of all infinite subsets of $\mathbb{N}$, and if $V$ is a Banach space with a normalized, 1-unconditional basis $\left(v_{i}\right)$, and if $N \in \mathbb{N}^{(\omega)}$, then we write $V_{N}$ for the closed linear span of $\left\{v_{i}: i \in N\right\}$. When we talk about subsequential $V_{N}$-lower estimates, etc., it is with respect to the normalized, 1-unconditional basis $\left(v_{i}\right)_{i \in N}$ of $V_{N}$.

Remark. Theorem 12 has a quantitative version. Let $C, D \in[1, \infty)$ and assume that $\left(v_{i}\right)$ is $D$-left-dominant and that $X$ satisfies subsequential $C$ - $V$-lower tree estimates.

Then for all $K \in[1, \infty)$ there is a constant $M=M(C, D, K) \in[1, \infty)$ such that in part (a) if $K(E, Z) \leq K$, then in the conclusion $X M$-embeds into $Z^{V_{N}}(H)$. Indeed, this follows directly from the proof. What is important is that $M$ depends only on the constants $C, D$ and $K$.

Also, there exists a constant $L=L(C, D) \in[1, \infty)$ such that in the conclusion of part (b) we get an onto map $\tilde{Q}: \tilde{Y}^{V_{N}}(\tilde{G}) \rightarrow X$ with $\|\tilde{Q}\|=1$ and $\tilde{Q}\left(L \cdot B_{\tilde{Y} V_{N}}\right) \supset B_{X}$. This also follows directly from the proof. However, the proof of part (b) uses [10, Lemma 3.1], which in turn appeals to a theorem of Zippin [18. The theorem of Zippin we need here states that every separable, reflexive space embeds isometrically into a reflexive space with an FDD. A quantitative version of this result claims 
the existence of a universal constant $K$ such that every separable, reflexive space embeds isometrically into a reflexive space with an FDD whose projection constant is at most $K$. Indeed, if this wasn't true, then for all $n \in \mathbb{N}$ there would be a "bad" space $X_{n}$, and then the $\ell_{2}$-sum of the sequence $\left(X_{n}\right)$ would contradict Zippin's theorem. The existence of this universal constant $K$ gives a quantitative version of (a special case of) 10, Lemma 3.1]: there is a universal constant $\bar{K}$ such that every separable, reflexive space $X$ embeds isometrically into a reflexive space $Z$ with an FDD $E=\left(E_{i}\right)$ with $K(E, Z) \leq \bar{K}$ such that $\mathrm{c}_{00}\left(\oplus_{i=1}^{\infty} E_{i}\right) \cap X$ is dense in $X$. The proof of part (b) now really does give the quantitative version of (b) stated above.

The consequences of all this are quantitative analogues of Corollaries 13 and 14 . and of Theorem 15. We shall state (without proof) the quantitative analogue of Theorem 15, and leave the reader to formulate the analogues of Corollaries 13 and 14. The proofs are straightforward: one simply needs to keep track of the various constants in the proofs of the qualitative statements.

Corollary 13. Assume that $V$ is a reflexive Banach space with a normalized and 1-unconditional basis $\left(v_{i}\right)$, and that $\left(v_{i}\right)$ is left-dominant and block-stable. Let $X$ be a separable, infinite-dimensional, reflexive space with subsequential $V$-lower tree estimates.

Then $X$ is a subspace of a reflexive space $Z$ with an FDD satisfying subsequential $V$-lower estimates and it is a quotient of a reflexive space $Y$ with an FDD satisfying subsequential $V$-lower estimates.

Proof. By a theorem of Zippin [18] we can embed $X$ into a reflexive space $W$ with an FDD $E=\left(E_{i}\right)$. Using Theorem 12 (a) we find a blocking $F=\left(F_{i}\right)$ of $\left(E_{i}\right)$ and $L \in \mathbb{N}^{(\omega)}$ such that $X$ embeds into $Z=W^{V_{L}}(F)$.

Theorem 12(b) provides a space $\tilde{Y}$ with a shrinking FDD $\tilde{G}=\left(\tilde{G}_{i}\right)$ and $M \in \mathbb{N}^{(\omega)}$ such that $X$ is a quotient of $Y=\tilde{Y}^{V_{M}}(\tilde{G})$.

By Corollary 9 the spaces $Z$ and $Y$ are reflexive. It follows from Lemma 10 that $\left(F_{i}\right)$ satisfies subsequential $V_{L}$-lower estimates in $Z$, and that $\left(\tilde{G}_{i}\right)$ satisfies subsequential $V_{M}$-lower estimates in $Y$. The result now follows from Lemma 2 (with $\left(u_{i}\right)$ the unit vector basis of $U=\ell_{1}$ ).

From Corollary 13 and Proposition 3 we deduce in certain instances the inverse implication of Proposition 4.

Corollary 14. Assume that $V$ is a reflexive Banach space with a normalized, 1 unconditional basis $\left(v_{i}\right)$, and that $\left(v_{i}\right)$ is left-dominant and block-stable.

If $X$ is a separable, infinite-dimensional, reflexive space which satisfies subsequential $V$-lower tree estimates, then $X^{*}$ satisfies subsequential $V^{*}$-upper tree estimates.

Proof. By Corollary $13 X$ is a quotient of a reflexive space with an FDD satisfying subsequential $V$-lower estimates. Hence, by Proposition $3 X^{*}$ is the subspace of a reflexive space $Z$ with an FDD $\left(E_{i}\right)$ satisfying subsequential $V^{*}$-upper estimates.

Now let $\left(x_{\alpha}\right)_{\alpha \in T_{\infty}^{\text {even }}}$ be a normalized, weakly null even tree in $X^{*}$. One can recursively choose $n_{1}<n_{2}<\ldots$ in $\mathbb{N}$ such that

$$
\left\|x_{\left(n_{1}, n_{2}, \ldots, n_{2 i}\right)}-P_{\left[n_{2 i-1}, n_{2 i+1}\right)}^{E}\left(x_{\left(n_{1}, n_{2}, \ldots, n_{2 i}\right)}\right)\right\|<2^{-i} \quad \text { for all } i \in \mathbb{N} .
$$

Set

$$
z_{i}=\frac{P_{\left[n_{2 i-1}, n_{2 i+1}\right)}^{E}\left(x_{\left(n_{1}, n_{2}, \ldots, n_{2 i}\right)}\right)}{\left\|P_{\left[n_{2 i-1}, n_{2 i+1}\right)}^{E}\left(x_{\left(n_{1}, n_{2}, \ldots, n_{2 i}\right)}\right)\right\|} \quad \text { for all } i \in \mathbb{N} .
$$

Then $\left(z_{i}\right)$ is dominated by $\left(v_{n_{2 i-1}}^{*}\right)$ since $\left(E_{i}\right)$ satisfies subsequential $V^{*}$-upper estimates. It follows that $\left(x_{\left(n_{1}, n_{2}, \ldots, n_{2 i}\right)}\right)$ is also dominated by $\left(v_{n_{2 i-1}}^{*}\right)$. 
Theorem 15. Let $V$ and $U$ be reflexive Banach spaces with 1-unconditional, normalized and block-stable bases $\left(v_{i}\right)$ and $\left(u_{i}\right)$, respectively. Further assume that $\left(v_{i}\right)$ is left-dominant, $\left(u_{i}\right)$ is right-dominant, and that $\left(v_{i}\right)$ is dominated by $\left(u_{i}\right)$.

If $X$ is a separable, infinite-dimensional, reflexive Banach space which satisfies subsequential $(V, U)$-tree estimates, then $X$ can be embedded into a reflexive Banach space $Z$ with an FDD $\left(G_{i}\right)$ which satisfies subsequential $(V, U)$-estimates in $Z$.

Proof. By Proposition $4 X^{*}$ satisfies subsequential $U^{*}$-lower tree estimates, and we can apply Corollary 13 to deduce that $X^{*}$ is the quotient of a reflexive space $Y^{*}$ with an FDD $\left(E_{i}^{*}\right)\left(Y^{*}\right.$ being the dual of a space $Y$ with an FDD $\left.\left(E_{i}\right)\right)$ satisfying subsequential $U^{*}$-lower estimates in $Y^{*}$. Thus $X$ is a subspace of the reflexive space $Y$ having an FDD $\left(E_{i}\right)$ which, by Proposition 3. satisfies subsequential $U$ upper estimates in $Y$.

Theorem 12 part (a) yields a blocking $F=\left(F_{i}\right)$ of $\left(E_{i}\right)$ and an infinite subset $M$ of $\mathbb{N}$ such that $X$ embeds into $Z=Y^{V_{M}}(F)$.

By Corollary 9 the space $Z$ is reflexive, and by Lemma $10\left(F_{i}\right)$ satisfies subsequential $V_{M}$-lower estimates in $Z$. Since $\left(E_{i}\right)$ satisfies subsequential $U$-upper estimates in $Y$, there exists $N \in \mathbb{N}^{(\omega)}$ such that $\left(F_{i}\right)$ satisfies subsequential $U_{N^{-}}$ upper estimates in $Y$. Since $\left(u_{i}\right)$ is right-dominant, we may assume after replacing $N$ if necessary that $m_{i} \leq n_{i}$ for all $i \in \mathbb{N}$, where $m_{i}$ and $n_{i}$ are the $i^{\text {th }}$ elements of $M$ and $N$, respectively. Now $\left(v_{i}\right)_{i \in M}$ is dominated by $\left(u_{i}\right)_{i \in N}$, so by Lemma 11 $\left(F_{i}\right)$ also satisfies subsequential $U_{N}$-upper estimates in $Z$. Finally, since $\left(v_{i}\right)$ is leftdominant, $\left(F_{i}\right)$ satisfies subsequential $\left(V_{N}, U_{N}\right)$ estimates in $Z$. An application of Lemma 2 completes the argument.

Before proceeding to the proof of Theorem 12 we state the quantitative version of Theorem [15] as promised earlier.

Theorem 16. For all $B, C, D, L, R \in[1, \infty)$ there exist constants $\bar{C}=\bar{C}(B, D, R)$ and $K=K(C, L, R)$ in $[1, \infty)$ such that the following holds. Let $V$ and $U$ be reflexive Banach spaces with 1-unconditional, normalized and B-block-stable bases $\left(v_{i}\right)$ and $\left(u_{i}\right)$, respectively. Further assume that $\left(v_{i}\right)$ is L-left-dominant, $\left(u_{i}\right)$ is $R$-right-dominant, and that $\left(v_{i}\right)$ is D-dominated by $\left(u_{i}\right)$.

If $X$ is a separable, infinite-dimensional, reflexive Banach space which satisfies subsequential $C$ - $(V, U)$-tree estimates, then $X$ can be $K$-embedded into a reflexive Banach space $Z$ which has a bimonotone $F D D\left(G_{i}\right)$ satisfying subsequential $\bar{C}$ $(V, U)$-estimates in $Z$.

Proof of Theorem 12 part (a). Choose constants $C$ and $D$ in $[1, \infty)$ such that $X$ satisfies subsequential $C$ - $V$-lower tree estimates and $\left(v_{i}\right)$ is $D$-left-dominant. Let $K$ be the projection constant of $\left(E_{i}\right)$ in $Z$. Set

$$
\mathcal{A}=\left\{\left(k_{i}, x_{i}\right) \in\left(\mathbb{N} \times S_{X}\right)^{\omega}:\left(v_{k_{i}}\right) \text { is } C \text {-dominated by }\left(x_{i}\right)\right\},
$$

and choose $\varepsilon>0$ such that

$$
\overline{\mathcal{A}_{\varepsilon}} \subset\left\{\left(k_{i}, x_{i}\right) \in\left(\mathbb{N} \times S_{X}\right)^{\omega}:\left(v_{k_{i}}\right) \text { is } 2 C D \text {-dominated by }\left(x_{i}\right)\right\} .
$$

By Proposition $\left[5\right.$ there exist $\left(K_{i}\right) \subset \mathbb{N}$ with $K_{1}<K_{2}<\ldots, \bar{\delta}=\left(\delta_{i}\right) \subset(0,1)$ with $\delta_{i} \downarrow 0$, and a blocking $F=\left(F_{i}\right)$ of $\left(E_{i}\right)$ such that if $\left(x_{i}\right) \subset S_{X}$ is a $(2 K \bar{\delta})$-skipped block sequence of $\left(F_{n}\right)$ in $Z$ with $\left\|x_{i}-P_{\left(r_{i-1}, r_{i}\right)}^{F} x_{i}\right\|<2 K \delta_{i}$ for all $i \in \mathbb{N}$, where $1 \leq r_{0}<r_{1}<r_{2}<\ldots$, then $\left(v_{K_{r_{i-1}}}\right)$ is $2 C D$-dominated by $\left(x_{i}\right)$.

It is easy to see that we can block $\left(F_{i}\right)$ into an FDD $G=\left(G_{i}\right)$ such that there exists $\left(e_{n}\right) \subset S_{X}$ with

$$
\left\|e_{n}-P_{n}^{G}\left(e_{n}\right)\right\|<\delta_{n} / 2 K \quad \text { for all } n \in \mathbb{N} .
$$


Let $\left(v_{i}^{\prime \prime}\right)$ be a subsequence of $\left(v_{i}\right)$ such that if $\left(x_{i}\right) \subset S_{X}$ is a $\bar{\delta}$-skipped block sequence of $\left(G_{n}\right)$ in $Z$ with $\left\|x_{i}-P_{\left(r_{i-1}, r_{i}\right)}^{G} x_{i}\right\|<\delta_{i}$ for all $i \in \mathbb{N}$, where $1 \leq r_{0}<r_{1}<r_{2}<\ldots$, then $\left(v_{r_{i-1}}^{\prime \prime}\right)$ is $2 C D$-dominated by $\left(x_{i}\right)$. Note that if $G_{j}=\bigoplus_{i=m_{j-1}+1}^{m_{j}} F_{i}, j \in \mathbb{N}, 0=$ $m_{0}<m_{1}<m_{2}<\ldots$, then $\left(v_{i}^{\prime \prime}\right)=\left(v_{K_{m_{i}}}\right)$ will do.

In order to continue we need the following result from [10, which is due (in a different form) to W. B. Johnson [6].

Proposition 17. Let $X$ be a Banach space which is a subspace of a reflexive space $Z$ with an FDD $A=\left(A_{i}\right)$ having projection constant $K$. Let $\bar{\eta}=\left(\eta_{i}\right) \subset(0,1)$ with $\eta_{i} \downarrow 0$. Then there exist positive integers $N_{1}<N_{2}<\ldots$ such that the following holds. Given positive integers $1 \leq k_{0}<k_{1}<\ldots$ and $x \in S_{X}$, there exist $x_{i} \in X$ and $t_{i} \in\left(N_{k_{i-1}-1}, N_{k_{i-1}}\right)\left(i \in \mathbb{N}, N_{0}=0\right)$ such that

(a) $x=\sum_{i=1}^{\infty} x_{i}$, and for all $i \in \mathbb{N}$ we have (putting $t_{0}=0$ )

(b) either $\left\|x_{i}\right\|<\eta_{i}$ or $\left\|x_{i}-P_{\left(t_{i-1}, t_{i}\right)}^{A} x_{i}\right\|<\eta_{i}\left\|x_{i}\right\|$,

(c) $\left\|x_{i}-P_{\left(t_{i-1}, t_{i}\right)}^{A} x\right\|<\eta_{i}$,

(d) $\left\|x_{i}\right\|<K+1$,

(e) $\left\|P_{t_{i}}^{A} x\right\|<\eta_{i}$.

This result is in fact a slight variation of (and follows easily from the proof of) Corollary 4.4 in 10 .

We now apply Proposition 17 with $A=G$ and $\bar{\eta}=\bar{\delta}$ to obtain an appropriate sequence $N_{1}<N_{2}<\ldots$ of positive integers. Set $H_{j}=\bigoplus_{i=N_{j-1}+1}^{N_{j}} G_{i}$ for each $j \in \mathbb{N}$ (and with $N_{0}=0$ ), and let $\left(v_{i}^{\prime}\right)$ be the subsequence of $\left(v_{i}\right)$ defined by $v_{i}^{\prime}=v_{N_{i}}^{\prime \prime}$ for all $i \in \mathbb{N}$. Let $N \in \mathbb{N}^{(\omega)}$ be chosen such that $\left(v_{i}\right)_{i \in N}$ is the subsequence $\left(v_{i}^{\prime}\right)$ of $\left(v_{i}\right)$.

Fix $x \in S_{X}$ and a sequence $1 \leq n_{0}<n_{1}<\ldots$ in $\mathbb{N}$. We will show that

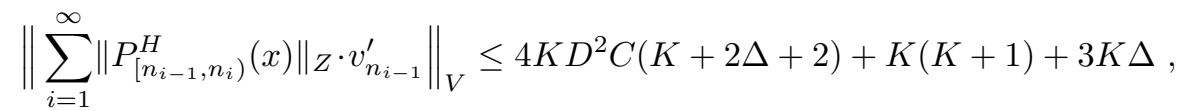

where $\Delta=\sum_{i=1}^{\infty} \delta_{i}$. Taking then the supremum over all choices of $\left(n_{i}\right)$, we obtain that the norms $\|\cdot\|_{Z}$ and $\|\cdot\|_{Z^{V_{N}(H)}}$ are equivalent when restricted to $X$, and hence statement (a) follows.

Set $M_{i}=N_{n_{i}-1}$ for $i=0,1,2, \ldots$. We thus have to show that

$$
\left\|\sum_{i=1}^{\infty}\right\| P_{\left(M_{i-1}, M_{i}\right]}^{G}(x)\left\|_{Z} \cdot v_{n_{i-1}}^{\prime}\right\|_{V} \leq 4 K D^{2} C(K+2 \Delta+2)+K(K+1)+3 K \Delta .
$$

For each $i \in \mathbb{N}$ choose $x_{i} \in X$ and $t_{i} \in\left(M_{i-1}, N_{n_{i-1}}\right)$ such that (a)-(e) of Proposition 17 hold with $A=G$ and $\bar{\eta}=\bar{\delta}$.

For each $i \in \mathbb{N}$ let $\bar{x}_{i}=\frac{x_{i+1}}{\left\|x_{i+1}\right\|}$ and $\alpha_{i}=\left\|x_{i+1}\right\|$ if $\left\|x_{i+1}\right\| \geq \delta_{i+1}$, and let $\bar{x}_{i}=e_{M_{i}}$ and $\alpha_{i}=0$ if $\left\|x_{i+1}\right\|<\delta_{i+1}$. Observe that $\left\|\bar{x}_{i}-P_{\left(t_{i}, t_{i+1}\right)}^{G}\left(\bar{x}_{i}\right)\right\|<\delta_{i}$ for all $i \in \mathbb{N}$, from which it follows that $\left(v_{t_{i}}^{\prime \prime}\right)$ is $2 C D$-dominated by $\left(\bar{x}_{i}\right)$. Hence

$$
\begin{aligned}
\left\|\sum_{i=1}^{\infty} x_{i}\right\|_{Z} & \geq\left\|\sum_{i=1}^{\infty} \alpha_{i} \bar{x}_{i}\right\|_{Z}-\left\|x_{1}\right\|_{Z}-\Delta \\
& \geq \frac{1}{2 C D}\left\|\sum_{i=1}^{\infty} \alpha_{i} v_{t_{i}}^{\prime \prime}\right\|_{V}-(K+1)-\Delta \\
& \geq \frac{1}{2 C D}\left\|\sum_{i=1}^{\infty}\right\| x_{i+1}\left\|_{Z} \cdot v_{t_{i}}^{\prime \prime}\right\|_{V}-\frac{1}{2 C D} \Delta-(K+1)-\Delta,
\end{aligned}
$$


and thus

$$
\left\|\sum_{i=1}^{\infty}\right\| x_{i+1}\left\|_{Z} \cdot v_{t_{i}}^{\prime \prime}\right\|_{V} \leq 2 C D(K+2 \Delta+2) .
$$

For each $i \in \mathbb{N}$ we have (putting $t_{0}=0$ )

$$
\left\|P_{\left(M_{i-1}, M_{i}\right]}^{G}(x)\right\|_{Z} \leq K\left\|P_{\left(t_{i-1}, t_{i+1}\right)}^{G}(x)\right\|_{Z} \leq K\left(\left\|x_{i}\right\|_{Z}+\left\|x_{i+1}\right\|_{Z}+3 \delta_{i}\right) .
$$

It follows that

$$
\begin{aligned}
& \left\|\sum_{i=1}^{\infty}\right\| P_{\left(M_{i-1}, M_{i}\right]}^{G}(x)\left\|_{Z} \cdot v_{n_{i-1}}^{\prime}\right\|_{V} \\
& \quad \leq K\left\|\sum_{i=1}^{\infty}\right\| x_{i}\left\|_{Z} \cdot v_{n_{i-1}}^{\prime}\right\|_{V}+K\left\|\sum_{i=1}^{\infty}\right\| x_{i+1}\left\|_{Z} \cdot v_{n_{i-1}}^{\prime}\right\|_{V}+3 K \Delta \\
& \quad \leq K\left\|\sum_{i=1}^{\infty}\right\| x_{i+1}\left\|_{Z} \cdot v_{n_{i}}^{\prime}\right\|_{V}+K\left\|\sum_{i=1}^{\infty}\right\| x_{i+1}\left\|_{Z} \cdot v_{n_{i-1}}^{\prime}\right\|_{V}+K(K+1)+3 K \Delta \\
& \leq 2 K D\left\|\sum_{i=1}^{\infty}\right\| x_{i+1}\left\|_{Z} \cdot v_{t_{i}}^{\prime \prime}\right\|_{V}+K(K+1)+3 K \Delta
\end{aligned}
$$

(since $v_{n_{i-1}}^{\prime}=v_{N_{n_{i-1}}}^{\prime \prime}$ and $N_{n_{i-1}}>t_{i}$ for all $i \in \mathbb{N}$ )

$$
\leq 4 K D^{2} C(K+2 \Delta+2)+K(K+1)+3 K \Delta
$$

Before we prove part (b) of Theorem 12 we need a blocking result due to Johnson and Zippin.

Proposition 18. 7] Let $T: Y \rightarrow Z$ be a bounded linear operator from a space $Y$ with a shrinking FDD $\left(G_{i}\right)$ into a space $Z$ with an FDD $\left(H_{i}\right)$. Let $\varepsilon_{i} \downarrow 0$. Then there exist blockings $E=\left(E_{i}\right)$ of $\left(G_{i}\right)$ and $F=\left(F_{i}\right)$ of $\left(H_{i}\right)$ so that for all $m<n$ and $y \in S_{\oplus_{i \in(m, n)} E_{i}}$ we have $\left\|P_{[1, m)}^{F} T y\right\|<\varepsilon_{m}$ and $\left\|P_{[n, \infty)}^{F} T y\right\|<\varepsilon_{n}$.

Proof of Theorem 12 part (b). By Lemma 3.1 in [10] we can, after renorming $X$ if necessary, regard $X^{*}$ (isometrically) as a subspace of a reflexive space $Y^{*}$ (being the dual of a reflexive space $Y$ with bimonotone FDD $\left.\left(E_{i}\right)\right)$ such that $\mathrm{c}_{00}\left(\oplus_{i=1}^{\infty} E_{i}^{*}\right) \cap X^{*}$ is dense in $X^{*}$. We have a natural quotient map $Q: Y \rightarrow X$. By a theorem of Zippin [18] we may regard $X$ (isometrically) as a subspace of a reflexive space $Z$ with an FDD $\left(F_{i}^{\prime}\right)$. Let $K$ be the projection constant of $\left(F_{i}^{\prime}\right)$ in $Z$, and choose constants $C$ and $D$ in $[1, \infty)$ such that $X$ satisfies subsequential $C$ - $V$-lower tree estimates and $\left(v_{i}\right)$ is $D$-left-dominant.

Using Proposition 5 as in the proof of part (a), we find sequences $\left(K_{i}\right) \subset \mathbb{N}$ with $K_{1}<K_{2}<\ldots, \bar{\delta}=\left(\delta_{i}\right) \subset(0,1)$ with $\delta_{i} \downarrow 0$, and a blocking $\left(F_{i}\right)$ of $\left(F_{i}^{\prime}\right)$ such that if $\left(x_{i}\right) \subset S_{X}$ is a $2 K \bar{\delta}$-skipped block sequence of $\left(F_{n}\right)$ in $Z$ with $\left\|x_{i}-P_{\left(r_{i-1}, r_{i}\right)}^{F} x_{i}\right\|_{Z}<$ $2 K \delta_{i}$ for all $i \in \mathbb{N}$, where $1 \leq r_{0}<r_{1}<r_{2}<\ldots$, then $\left(v_{K_{r_{i-1}}}\right)$ is $2 C D$-dominated by $\left(x_{i}\right)$, and moreover, using standard perturbation arguments and making $\bar{\delta}$ smaller if necessary, we can assume that if $\left(z_{i}\right) \subset Z$ satisfies $\left\|x_{i}-z_{i}\right\|_{Z}<\delta_{i}$ for all $i \in \mathbb{N}$, then $\left(z_{i}\right)$ is a basic sequence equivalent to $\left(x_{i}\right)$ with projection constant at most $2 K$. We also require that

$$
\Delta=\sum_{i=1}^{\infty} \delta_{i}<\frac{1}{7}
$$


Choose a sequence $\bar{\varepsilon}=\left(\varepsilon_{i}\right) \subset(0,1)$ with $\varepsilon_{i} \downarrow 0$ and

$$
3 K(K+1) \sum_{j=i}^{\infty} \varepsilon_{j}<\delta_{i}^{2} \quad \text { for all } i \in \mathbb{N} .
$$

After blocking $\left(F_{i}\right)$ if necessary, we can assume that for any subsequent blocking $D$ of $F$ there is a sequence $\left(e_{i}\right)$ in $S_{X}$ such that

$$
\left\|e_{i}-P_{i}^{D}\left(e_{i}\right)\right\|_{Z}<\varepsilon_{i} / 2 K \quad \text { for all } i \in \mathbb{N} .
$$

By Proposition 18 we may assume, after further blocking our FDDs if necessary, that

$$
\begin{aligned}
& \text { for all } m<n \text { and } y \in S_{\oplus_{i \in(m, n)} E_{i}} \text { we have } \\
& \qquad\left\|P_{[1, m)}^{F} \circ Q(y)\right\|<\varepsilon_{m} \quad \text { and } \quad\left\|P_{[n, \infty)}^{F} \circ Q(y)\right\|<\varepsilon_{n},
\end{aligned}
$$

and moreover the same holds if one passes to any blocking of $\left(E_{i}\right)$ and the corresponding blocking of $\left(F_{i}\right)$.

For $i \in \mathbb{N}$ let $\tilde{E}_{i}$ be the quotient space of $E_{i}$ determined by $Q$, i.e., if $y \in E_{i}$, then the norm of $\tilde{y}$, the equivalence class of $y$ in $E_{i}$, is given by $\|\tilde{y}\|=\|Q(y)\|$. Passing to a further blocking of $\left(E_{i}\right)$ (and the corresponding blocking of $\left(F_{i}\right)$ ), we may assume that $\tilde{E}_{i} \neq\{0\}$ for all $i \in \mathbb{N}$. Given $y=\sum y_{i} \in \mathrm{c}_{00}\left(\oplus_{i=1}^{\infty} E_{i}\right), y_{i} \in E_{i}$ for all $i \in \mathbb{N}$, we set $\tilde{y}=\sum \tilde{y}_{i} \in \mathrm{c}_{00}\left(\oplus_{i=1}^{\infty} \tilde{E}_{i}\right)$ and

$$
\|\tilde{y}\|=\max _{m<n}\left\|\sum_{i=m}^{n} Q\left(y_{i}\right)\right\|=\max _{m<n}\left\|Q \circ P_{[m, n]}^{E}(y)\right\| .
$$

We let $\tilde{Y}$ be the completion of $\mathrm{c}_{00}\left(\oplus_{i=1}^{\infty} \tilde{E}_{i}\right)$ with respect to $\|\cdot \cdot\| \cdot$. Since $\left(E_{i}\right)$ is a bimonotone FDD in $Y$, we have $\|\tilde{y}\| \leq\|y\|$ for all $y \in \mathrm{c}_{00}\left(\oplus_{i=1}^{\infty} E_{i}\right)$, and hence the map $y \mapsto \tilde{y}$ extends to a norm one map from $Y$ to $\tilde{Y}$. By the definition of $\||\cdot| \mid$ we have $\|Q y\| \leq\|\tilde{y}\|$ for any $y \in \mathrm{c}_{00}\left(\oplus_{i=1}^{\infty} E_{i}\right)$. It follows that $\tilde{y} \mapsto Q(y)$ extends to a norm one map $\tilde{Q}: \tilde{Y} \rightarrow X$ with $\tilde{Q}(\tilde{y})=Q(y)$ for all $y \in Y$.

In order to continue our proof we will need the following proposition from [1].

Proposition 19. [11, Proposition 2.6]

(a) $\left(\tilde{E}_{i}\right)$ is a bimonotone, shrinking FDD for $\tilde{Y}$.

(b) $\tilde{Q}$ is a quotient map from $\tilde{Y}$ onto $X$. More precisely if $x \in X$ and $y \in Y$ is such that $Q(y)=x,\|y\|=\|x\|$ and $y=\sum y_{i}$ with $y_{i} \in E_{i}$ for all $i \in \mathbb{N}$, then $\tilde{y}=\sum \tilde{y}_{i} \in \tilde{Y},\|\tilde{y}\|=\|y\|$ and $\tilde{Q}(\tilde{y})=x$.

(c) Let $\left(\tilde{y}_{i}\right)$ be a block sequence of $\left(\tilde{E}_{n}\right)$ in $B_{\tilde{Y}}$, and assume that $\left(\tilde{Q}\left(\tilde{y}_{i}\right)\right)$ is a basic sequence with projection constant $\bar{K}$ and that $a=\inf _{i}\left\|\tilde{Q}\left(\tilde{y}_{i}\right)\right\|>0$. Then for all $\left(a_{i}\right) \in \mathrm{c}_{00}$ we have

$$
\left\|\sum a_{i} \tilde{Q}\left(\tilde{y}_{i}\right)\right\| \leq\left\|\sum a_{i} \tilde{y}_{i}\right\| \leq \frac{3 \bar{K}}{a}\left\|\sum a_{i} \tilde{Q}\left(\tilde{y}_{i}\right)\right\| .
$$

To finish the proof of Theorem 12 (b) it suffices to find a constant $L<\infty$, a subsequence $\left(v_{i}^{\prime}\right)$ of $\left(v_{i}\right)$, and a blocking $\tilde{G}=\left(\tilde{G}_{i}\right)$ of $\left(\tilde{E}_{i}\right)$ with the following property. For each $x \in S_{X}$ there exists a $\tilde{y}=\sum \tilde{y}_{i} \in \tilde{Y}, \tilde{y}_{i} \in \tilde{G}_{i}$ for all $i \in \mathbb{N}$, such that

$$
\begin{aligned}
& \|\tilde{Q}(\tilde{y})-x\|<1 / 2, \\
& \left\|\sum_{j=1}^{\infty}\right\| P_{\left[n_{j-1}, n_{j}\right)}^{\tilde{\tilde{y}}}(\tilde{y})\left\|\cdot v_{n_{j-1}}^{\prime}\right\|_{V} \leq L
\end{aligned}
$$

for any choice of $k$ and $1 \leq n_{0}<n_{1}<n_{2}<\ldots$ in $\mathbb{N}$. 
Once this is accomplished, we consider the space $\tilde{Y}^{V_{N}}=\tilde{Y}^{V_{N}}(\tilde{G})$, where $N \in \mathbb{N}^{(\omega)}$ is chosen so that $\left(v_{i}\right)_{i \in N}$ is the subsequence $\left(v_{i}^{\prime}\right)$ of $\left(v_{i}\right)$. Given $x=x_{0} \in S_{X}$, the property of $\tilde{G}$ allows us to recursively choose $x_{n} \in \frac{1}{2^{n}} B_{X}$ and $\tilde{y}_{n} \in \frac{L}{2^{n-1}} B_{\tilde{Y} V_{N}}, n \in \mathbb{N}$, so that $x_{n}=x_{n-1}-\tilde{Q}\left(\tilde{y}_{n}\right)$ for all $n \in \mathbb{N}$. It follows that $\sum_{n=1}^{\infty} \tilde{y}_{n}$ converges in $\tilde{Y}^{V_{N}}$ with $\left\|\sum_{n=1}^{\infty} \tilde{y}_{n}\right\|_{\tilde{Y} V_{N}} \leq 2 L$ and $\tilde{Q}\left(\sum_{n=1}^{\infty} \tilde{y}_{n}\right)=x$. Thus $\tilde{Q}: \tilde{Y}^{V_{N}} \rightarrow X$ remains surjective, which finishes the proof.

In order to show the existence of a suitable blocking $\tilde{G}$ of $\tilde{E}$ we need the following result from [11.

Lemma 20. [11, Lemma 2.7] Assume that (13) holds for our original map $Q: Y \rightarrow$ $X$. Then there exist integers $0=N_{0}<N_{1}<\ldots$ so that if for each $i \in \mathbb{N}$ we define

$$
\begin{aligned}
& C_{i}=\bigoplus_{j=N_{i-1}+1}^{N_{i}} E_{j}, \quad D_{i}=\bigoplus_{j=N_{i-1}+1}^{N_{i}} F_{j}, \\
& L_{i}=\left\{j \in \mathbb{N}: N_{i-1}<j \leq \frac{N_{i-1}+N_{i}}{2}\right\}, \\
& R_{i}=\left\{j \in \mathbb{N}: \frac{N_{i-1}+N_{i}}{2}<j \leq N_{i}\right\}, \\
& C_{i, L}=\bigoplus_{j \in L_{i}} E_{j} \quad \text { and } \quad C_{i, R}=\bigoplus_{j \in R_{i}} E_{j},
\end{aligned}
$$

then the following holds. Let $x \in S_{X}, 0 \leq m<n$ and $\varepsilon>0$, and assume that $\left\|x-P_{(m, n)}^{D}(x)\right\|<\varepsilon$. Then there exists $y \in B_{Y}$ with $y \in C_{m, R} \oplus\left(\bigoplus_{i \in(m, n)} C_{i}\right) \oplus C_{n, L}$ (where $C_{0, R}=\{0\}$ ) and $\|Q y-x\|<K\left(2 \varepsilon+\varepsilon_{m+1}\right)$ (recall that $K$ is the projection constant of $\left(F_{i}^{\prime}\right)$ in $\left.Z\right)$.

Let $\left(C_{i}\right)$ and $\left(D_{i}\right)$ be the blockings given by Lemma 20. Note that the sequence $\left(N_{i}\right)$ in the lemma used to define these blockings will not be needed in the sequel, so we can discard it. We now apply Proposition 17 with $\left(A_{i}\right)=\left(D_{i}\right)$ and $\bar{\eta}=\bar{\varepsilon}$ to obtain a sequence $N_{1}<N_{2}<\ldots$ in $\mathbb{N}$ so that the conclusions of the proposition are satisfied. Let $\left(v_{i}^{\prime \prime}\right)$ be a subsequence of $\left(v_{i}\right)$ such that if $\left(x_{i}\right) \subset S_{X}$ is a $\bar{\delta}$-skipped block sequence of $\left(D_{n}\right)$ in $Z$ with $\left\|x_{i}-P_{\left(r_{i-1}, r_{i}\right)}^{D} x_{i}\right\|_{Z}<\delta_{i}$ for all $i \in \mathbb{N}$, where $1 \leq r_{0}<r_{1}<r_{2}<\ldots$, then $\left(v_{r_{i-1}}^{\prime \prime}\right)$ is $2 C D$-dominated by $\left(x_{i}\right)$, and moreover, if $\left(z_{i}\right) \subset Z$ satisfies $\left\|x_{i}-z_{i}\right\|<\delta_{i}$ for all $i \in \mathbb{N}$, then $\left(z_{i}\right)$ is a basic sequence equivalent to $\left(x_{i}\right)$ with projection constant at most $2 K$. Let $\left(v_{i}^{\prime}\right)$ be the subsequence of $\left(v_{i}\right)$ defined by setting $v_{i}^{\prime}=v_{N_{i}}^{\prime \prime}$ for all $i \in \mathbb{N}$. We now come to our final blockings: for each $i \in \mathbb{N}$ set $G_{i}=\bigoplus_{j=N_{i-1}+1}^{N_{i}} C_{j}$ and let $H_{i}=\bigoplus_{j=N_{i-1}+1}^{N_{i}} D_{j}\left(N_{0}=0\right)$. Put $G=\left(G_{i}\right)$, let $\tilde{G}=\left(\tilde{G}_{i}\right)$ be the corresponding blocking of $\left(\tilde{E}_{i}\right)$, and set $H=\left(H_{i}\right)$.

Fix a sequence $\left(e_{i}\right)$ in $S_{X}$ so that (12) holds. Let $x \in S_{X}$. By the choice of $N_{1}, N_{2}, \ldots$, for each $i \in \mathbb{N}$, there are $x_{i} \in(K+1) B_{X}$ and $t_{i} \in\left(N_{i-1}, N_{i}\right)$ such that $x=\sum_{i=1}^{\infty} x_{i}$ and for all $i \in \mathbb{N}$ either $\left\|x_{i}\right\|<\varepsilon_{i}$ or $\left\|P_{\left(t_{i-1}, t_{i}\right)}^{D} x_{i}-x_{i}\right\|<\varepsilon_{i}\left\|x_{i}\right\|\left(t_{0}=0\right)$. For each $i \in \mathbb{N}$ let $\bar{x}_{i}=\frac{x_{i+1}}{\left\|x_{i+1}\right\|}$ and $\alpha_{i}=\left\|x_{i+1}\right\|$ if $\left\|x_{i+1}\right\| \geq \varepsilon_{i+1}$, and let $\bar{x}_{i}=e_{N_{i}}$ and $\alpha_{i}=0$ if $\left\|x_{i+1}\right\|<\varepsilon_{i+1}$.

Since

$$
\left\|\bar{x}_{i}-P_{\left(t_{i}, t_{i+1}\right)}^{D}\left(\bar{x}_{i}\right)\right\|<\varepsilon_{i+1} \quad \text { for all } i \in \mathbb{N},
$$

there exists $\left(y_{i}\right) \subset B_{Y}$ with $y_{i} \in C_{t_{i}, R} \oplus\left(\bigoplus_{j \in\left(t_{i}, t_{i+1}\right)} C_{j}\right) \oplus C_{t_{i+1}, L}$ and

$$
\left\|Q\left(y_{i}\right)-\bar{x}_{i}\right\|<3 K \varepsilon_{i+1}, \quad i \in \mathbb{N} .
$$

Also, if $\left\|x_{1}\right\|<\varepsilon_{1}$, then set $y_{0}=0$, and if $\left\|x_{1}\right\| \geq \varepsilon_{1}$, then choose $y_{0} \in(K+1) B_{Y}$ such that $y_{0} \in\left(\bigoplus_{j \in\left(0, t_{1}\right)} C_{j}\right) \oplus C_{t_{1}, L} \subset G_{1}$ and $\left\|Q\left(y_{0}\right)-x_{1}\right\|<3 K(K+1) \varepsilon_{1}$. 
Set $\bar{x}=x_{1}+\sum_{i=1}^{\infty} \alpha_{i} \bar{x}_{i}$, and note that (this series converges and) by (10) and (11)

$$
\|x-\bar{x}\| \leq \sum_{i=2}^{\infty} \varepsilon_{i}<\frac{1}{4} .
$$

As a $\bar{\delta}$-skipped block sequence of $\left(D_{i}\right)$ (this follows from (16) and (11)), $\left(\bar{x}_{i}\right)$ is a basic sequence with projection constant at most $2 K$ that $2 C D$-dominates $\left(v_{t_{i}}^{\prime \prime}\right)$. Since, by (17), $\left\|\tilde{Q}\left(\tilde{y}_{i}\right)-\bar{x}_{i}\right\|<3 K \varepsilon_{i+1}<\delta_{i}$ for all $i \in \mathbb{N}$, the sequence $\left(\tilde{Q}\left(\tilde{y}_{i}\right)\right)$ is also a basic sequence with projection constant at most $2 K$ and is equivalent to $\left(\bar{x}_{i}\right)$. Furthermore, we have $\inf _{i}\left\|\tilde{Q}\left(\tilde{y}_{i}\right)\right\| \geq \inf _{i}\left(\left\|\bar{x}_{i}\right\|-\delta_{i}\right)>6 / 7$, and thus, by Proposition 19 (c),

$$
\left\|\sum a_{i} \tilde{Q}\left(\tilde{y}_{i}\right)\right\| \leq\left\|\sum a_{i} \tilde{y}_{i}\right\| \leq x K\left\|\sum a_{i} \tilde{Q}\left(\tilde{y}_{i}\right)\right\| \quad \text { for all }\left(a_{i}\right) \in \mathrm{c}_{00} .
$$

Thus $\left(\tilde{y}_{i}\right)$ is a basic sequence equivalent to $\left(\bar{x}_{i}\right)$ and, in particular, $\sum_{i=1}^{\infty} \alpha_{i} \tilde{y}_{i}$ converges. Putting $\tilde{y}=\tilde{y}_{0}+\sum_{i=1}^{\infty} \alpha_{i} \tilde{y}_{i}$ we have

$$
\begin{aligned}
\|\tilde{Q} \tilde{y}-\bar{x}\| & \leq\left\|\tilde{Q} \tilde{y}_{0}-x_{1}\right\|+\sum_{i=1}^{\infty}\left|\alpha_{i}\right| \cdot\left\|\tilde{Q} \tilde{y}_{i}-\bar{x}_{i}\right\| \\
& \leq 3 K(K+1) \sum_{i=1}^{\infty} \varepsilon_{i}<1 / 4
\end{aligned}
$$

and hence, by (18), $\|\tilde{Q} \tilde{y}-x\|<1 / 2$, so we have (14).

We now fix integers $1 \leq n_{0}<n_{1}<n_{2}<\ldots$ We have $\tilde{y}_{i} \in \tilde{G}_{i} \oplus \tilde{G}_{i+1}$ for each $i \in \mathbb{N}$, and $\tilde{y}_{0} \in \tilde{G}_{1}$. It follows that

$$
\begin{aligned}
\left\|\sum_{s=1}^{\infty}\right\| P_{\left[n_{s-1}, n_{s}\right)}^{\tilde{G}}(\tilde{y})\left\|\cdot v_{n_{s-1}}^{\prime}\right\|_{V} \leq\left\|\tilde{y}_{0}\right\| & +\left\|\sum_{s=1}^{\infty} \alpha_{n_{s-1}-1} \cdot v_{n_{s-1}}^{\prime}\right\|_{V} \\
& +\left\|\sum_{s=1}^{\infty}\right\|\left\|\sum_{i=n_{s-1}}^{n_{s}-1} \alpha_{i} \tilde{y}_{i}\right\|\left\|_{n_{s-1}}^{\prime}\right\|_{V},
\end{aligned}
$$

where we put $\alpha_{0}=0$ in case $n_{0}=1$. We now show how to bound each of the three terms of the right-hand side of the above inequality, and hence obtain (15) with $L=126 C D^{2} K^{3}$.

We already have $\left\|\tilde{y}_{0}\right\| \leq K+1$. Since $\left(\bar{x}_{i}\right) 2 C D$-dominates $\left(v_{t_{i}}^{\prime \prime}\right)$ we get

$$
\begin{aligned}
2 C D^{2}\left\|\sum_{i} \alpha_{i} \bar{x}_{i}\right\|_{Z} & \geq D\left\|\sum_{i} \alpha_{i} \cdot v_{t_{i}}^{\prime \prime}\right\|_{V} \\
& \geq\left\|\sum_{i} \alpha_{i} \cdot v_{i+1}^{\prime}\right\|_{V}
\end{aligned}
$$

(since $v_{i+1}^{\prime}=v_{N_{i+1}}^{\prime \prime}$ and $N_{i+1}>t_{i}$ for all $i \in \mathbb{N}$ )

$$
\geq\left\|\sum_{s=1}^{\infty} \alpha_{n_{s-1}-1} \cdot v_{n_{s-1}}^{\prime}\right\|_{V} .
$$

Moreover, it follows from (18) that

$$
\left\|\sum_{i} \alpha_{i} \bar{x}_{i}\right\|_{Z}=\left\|\bar{x}-x_{1}\right\|_{Z} \leq K+3
$$

This yields the bound of $2 C D^{2}(K+3)$ for the second term of (20). 
For each $s \in \mathbb{N}$ let $\tilde{w}_{s}=\sum_{i=n_{s-1}}^{n_{s}-1} \alpha_{i} \tilde{y}_{i}$ and $b_{s}=\sum_{i=n_{s-1}}^{n_{s}-1} \alpha_{i} \bar{x}_{i}$. Note that by (16) and (11)

$\begin{aligned}\left\|b_{s}-P_{\left(t_{n_{s-1}}, t_{n_{s}}\right)}^{D}\left(b_{s}\right)\right\| & \leq \sum_{i=n_{s-1}}^{n_{s}-1}\left|\alpha_{i}\right| \cdot 2 K \cdot\left\|\bar{x}_{i}-P_{\left(t_{i}, t_{i+1}\right)}^{D} \bar{x}_{i}\right\| \\ & <2 K(K+1) \sum_{i=n_{s-1}}^{n_{s}-1} \varepsilon_{i+1}<\delta_{s}^{2} \quad \text { for all } s \in \mathbb{N} .\end{aligned}$

For each $s \in \mathbb{N}$ set $\bar{b}_{s}=\frac{b_{s}}{\left\|b_{s}\right\|}$ and $\beta_{s}=\left\|b_{s}\right\|$ if $\left\|b_{s}\right\| \geq \delta_{s}$, and set $\bar{b}_{s}=\bar{x}_{n_{s-1}}$ and $\beta_{s}=0$ if $\left\|b_{s}\right\|<\delta_{s}$. It follows from (22) and (16) that $\left(\bar{b}_{s}\right) \subset S_{X}$ is a $\bar{\delta}$-skipped block sequence of $\left(D_{i}\right)$ in $Z$ with $\left\|\bar{b}_{s}-P_{\left(t_{n_{s-1}}, t_{n_{s}}\right)}^{D}\left(\bar{b}_{s}\right)\right\|<\delta_{s}$ for all $s \in \mathbb{N}$, and hence it is a basic sequence that $2 C D$-dominates $\left(v_{t_{n_{s-1}}}^{\prime \prime}\right)$.

From (17) and (19) we have

$$
\begin{aligned}
\left\|\tilde{Q}\left(\tilde{w}_{s}\right)-b_{s}\right\| & \leq \sum_{i=n_{s-1}}^{n_{s}-1}\left|\alpha_{i}\right| \cdot\left\|\tilde{Q}\left(\tilde{y}_{i}\right)-\bar{x}_{i}\right\| \\
& <3 K(K+1) \sum_{i=n_{s-1}}^{n_{s}-1} \varepsilon_{i+1}<\delta_{s}
\end{aligned}
$$

and

$$
\left\|\tilde{w}_{s}\right\| \leq 7 K\left\|\tilde{Q}\left(\tilde{w}_{s}\right)\right\| \quad \text { for all } s \in \mathbb{N} .
$$

We now obtain the following sequence of inequalities.

$$
\begin{aligned}
\left\|\sum_{s=1}^{\infty}\right\| \tilde{w}_{s}\left\|\cdot v_{n_{s-1}}^{\prime}\right\|_{V} & \leq 7 K\left\|\sum_{s=1}^{\infty}\right\| \tilde{Q}\left(\tilde{w}_{s}\right)\left\|\cdot v_{n_{s-1}}^{\prime}\right\|_{V} \quad \text { (from (24)) } \\
& \leq 7 K\left\|\sum_{s=1}^{\infty}\right\| b_{s}\left\|\cdot v_{n_{s-1}}^{\prime}\right\|_{V}+7 K \Delta \quad \text { (from (23)) } \\
& \leq 7 K\left\|\sum_{s=1}^{\infty} \beta_{s} v_{n_{s-1}}^{\prime}\right\|_{V}+14 K \Delta \\
& \leq 7 K D\left\|\sum_{s=1}^{\infty} \beta_{s} v_{t_{n_{s-1}}^{\prime \prime}}\right\|_{V}+14 K \Delta
\end{aligned}
$$

(as $\left(v_{i}\right)$ is $D$-left-dominant)

$$
\leq 14 C D^{2} K\left\|\sum_{s=1}^{\infty} \beta_{s} \bar{b}_{s}\right\|+14 K \Delta
$$

(since $\left(\bar{b}_{s}\right) 2 C D$-dominates $\left.\left(v_{t_{n_{s-1}}}^{\prime \prime}\right)\right)$

$$
\leq 14 C D^{2} K\left\|\sum_{i=n_{0}}^{\infty} \alpha_{i} \bar{x}_{i}\right\|+14 C D^{2} K \Delta+14 K \Delta .
$$

Finally, since $\left(\bar{x}_{i}\right)$ is a basic sequence with projection constant at most $2 K$, it follows from (21) that

$$
\left\|\sum_{i=n_{0}}^{\infty} \alpha_{i} \bar{x}_{i}\right\|_{Z} \leq 2 K\left\|\sum_{i=1}^{\infty} \alpha_{i} \bar{x}_{i}\right\|_{Z} \leq 2 K(K+3)
$$


This provides an upper bound of $116 C D^{2} K^{3}$ for the third term of (20), which leads (15) with $L=126 C D^{2} K^{3}$, as claimed. This completes the proof of part (b) of Theorem 12 .

\section{UNIVERSAL CONSTRUCTIONS AND APPLICATIONS}

Let $V$ and $U$ be reflexive spaces with normalized, 1-unconditional, block-stable bases $\left(v_{i}\right)$ and $\left(u_{i}\right)$, respectively, such that $\left(v_{i}\right)$ is left-dominant, $\left(u_{i}\right)$ is rightdominant and $\left(v_{i}\right)$ is dominated by $\left(u_{i}\right)$. For each $C \in[1, \infty)$ let $\mathcal{A}_{V, U}(C)$ denote the class of all separable, infinite-dimensional, reflexive Banach spaces that satisfy subsequential $C$ - $(V, U)$-tree estimates. We also let

$$
\mathcal{A}_{V, U}=\bigcup_{C \in[1, \infty)} \mathcal{A}_{V, U}(C),
$$

which is the class of all separable, infinite-dimensional, reflexive Banach spaces that satisfy subsequential $(V, U)$-tree estimates.

Theorem 21. The class $\mathcal{A}_{V, U}$ defined above contains an element which is universal for the class.

More precisely, for all $B, D, L, R \in[1, \infty)$ there exists a constant $\bar{C}=\bar{C}(B, D) \in$ $[1, \infty)$ and for all $C \in[1, \infty)$ there is a constant $K(C)=K_{B, D, L, R}(C) \in[1, \infty)$ such that if $\left(v_{i}\right)$ is B-block-stable and L-left-dominant, if $\left(u_{i}\right)$ is B-block-stable and $R$ right-dominant, and if $\left(v_{i}\right)$ is D-dominated by $\left(u_{i}\right)$, then there exists $Z \in \mathcal{A}_{V, U}$ such that for all $C \in[1, \infty)$ every $X \in \mathcal{A}_{V, U}(C) K(C)$-embeds into $Z$, and moreover $Z$ has a bimonotone FDD satisfying subsequential $\bar{C}-(V, U)$ estimates in $Z$.

Proof. By a result of Schechtman [16] there exists a space $W$ with a bimonotone FDD $E=\left(E_{i}\right)$ with the property that any bimonotone FDD is naturally almost isometric to a subsequence $\overline{\bigoplus_{i=1}^{\infty} E_{k_{i}}}$ which is 1-complemented in $W$. More precisely, given a Banach space $X$ with a bimonotone FDD $\left(F_{i}\right)$ and given $\varepsilon>0$, there is a subsequence $\left(E_{k_{i}}\right)$ of $\left(E_{i}\right)$ and a $(1+\varepsilon)$-embedding $T: X \rightarrow W$ such that $T\left(F_{i}\right)=E_{k_{i}}$ for all $i \in \mathbb{N}$, and $\sum_{i=1}^{\infty} P_{k_{i}}^{E}$ is a norm-1 projection of $W$ onto $\overline{\bigoplus_{i=1}^{\infty} E_{k_{i}}}$.

We shall now modify the norm on $W$ in two stages. We first consider the space $\left(W^{(*)}\right)^{U^{*}}\left(E^{*}\right)$. By Corollary 7 the sequence $\left(E_{i}^{*}\right)$ is a boundedly complete (and bimonotone) FDD for this space. It follows that $\left(E_{i}\right)$ is a bimonotone, shrinking FDD for a space $Y$ with $Y^{*}=\left(W^{(*)}\right)^{U^{*}}\left(E^{*}\right)$. By Lemma 10 and Proposition 3 $\left(E_{i}\right)$ satisfies subsequential $2 B$ - $U$-upper estimates in $Y$.

We now let $Z=Y^{V}(E)$. By Corollary $9 Z$ is reflexive, by Lemma $10\left(E_{i}\right)$ satisfies subsequential $2 B$ - $V$-lower estimates in $Z$, and by Lemma $11\left(E_{i}\right)$ also satisfies subsequential $\left(3 B D+2 B^{2} D\right)$ - $U$-upper estimates in $Z$. Thus $\left(E_{i}\right)$ is a bimonotone FDD satisfying subsequential $\bar{C}$ - $(V, U)$-estimates in $Z$, where $\bar{C}=3 B D+2 B^{2} D$. It remains to show that $Z$ is universal for $\mathcal{A}_{V, U}$.

Let $C \in[1, \infty)$ and let $X \in \mathcal{A}_{V, U}(C)$. By Theorem 16 there exist constants $K_{1}=$ $K_{1}(B, D, R)$ and $K_{2}=K_{2}(C, L, R)$ in $[1, \infty)$ such that $X K_{2}$-embeds into a reflexive space $\tilde{X}$ which has a bimonotone FDD $\left(F_{i}\right)$ satisfying subsequential $K_{1}-(V, U)$ estimates in $\tilde{X}$. Now we can find a subsequence $\left(E_{k_{i}}\right)$ of $\left(E_{i}\right)$ and a 2 -embedding $T: \tilde{X} \rightarrow W$ such that $T\left(F_{i}\right)=E_{k_{i}}$ for all $i \in \mathbb{N}$ and $\sum_{i} P_{k_{i}}^{E}$ is a norm-1 projection of $W$ onto $\overline{\bigoplus_{i} E_{k_{i}}}$. It follows in particular that $\left(E_{k_{i}}\right)$ satisfies subsequential $2 K_{1^{-}}$ $(V, U)$ estimates in $W$, i.e., if $\left(w_{i}\right)$ is a normalized block sequence of $\left(E_{k_{n}}\right)$ in $W$ with $\min \operatorname{supp}_{E}\left(w_{i}\right)=k_{m_{i}}$ for all $i \in \mathbb{N}$, then $\left(w_{i}\right) 2 K_{1}$-dominates $\left(v_{m_{i}}\right)$ and is $2 K_{1}$-dominated by $\left(u_{m_{i}}\right)$. Hence by Proposition $3\left(E_{k_{i}}^{*}\right)$ satisfies subsequential $2 K_{1^{-}}\left(U^{*}, V^{*}\right)$ estimates in $W^{(*)}$. (Note that the dual of the subspace $\overline{\bigoplus_{i} E_{k_{i}}}$ of $W$ is naturally isometrically isomorphic to the subspace $\overline{\bigoplus_{i} E_{k_{i}}^{*}}$ of $W^{(*)}$.) We shall 
now use this to show that the norms $\|\cdot\|_{W},\|\cdot\|_{Y}$ and $\|\cdot\|_{Z}$ are all equivalent when restricted to $c_{00}\left(\oplus_{i} E_{k_{i}}\right)$, which implies that $\tilde{X}$ and hence also $X$ embed into $Z$.

Fix $w^{*} \in \mathrm{c}_{00}\left(\oplus_{i} E_{k_{i}}^{*}\right)$. Clearly we have $\left\|w^{*}\right\|_{W^{(*)}} \leq\left\|w^{*}\right\|_{Y^{*}}$. Choose $1 \leq m_{0}<$ $m_{1}<\ldots$ in $\mathbb{N}$ such that

$$
\left\|w^{*}\right\|_{Y^{*}}=\left\|\sum_{i=1}^{\infty}\right\| P_{\left[m_{i-1}, m_{i}\right)}^{E^{*}} w^{*}\left\|_{W^{(*)}} \cdot u_{m_{i-1}}^{*}\right\|_{U^{*}} .
$$

We may assume that $m_{0}=1$ and $P_{\left[m_{i-1}, m_{i}\right)}^{E^{*}} w^{*} \neq 0$ for all $i \in \mathbb{N}$. Then there exist $j_{1}<j_{2}<\ldots$ in $\mathbb{N}$ such that $k_{j_{i}}=\min \operatorname{supp}_{E^{*}} P_{\left[m_{i-1}, m_{i}\right)}^{E^{*}} w^{*}$ for all $i \in \mathbb{N}$. Since $\left(u_{i}^{*}\right)$ is $B$-block-stable and $R$-left-dominant, and since $\left(E_{k_{i}}^{*}\right)$ satisfies subsequential $2 K_{1}-U^{*}$-lower estimates in $W^{(*)}$, we have

$$
\begin{aligned}
\left\|w^{*}\right\|_{Y^{*}} & \leq B\left\|\sum_{i=1}^{\infty}\right\| P_{\left[m_{i-1}, m_{i}\right)}^{E^{*}} w^{*}\left\|_{W^{(*)}} \cdot u_{k_{j_{i}}}^{*}\right\|_{U^{*}} \\
& \leq B R\left\|\sum_{i=1}^{\infty}\right\| P_{\left[m_{i-1}, m_{i}\right)}^{E^{*}} w^{*}\left\|_{W^{(*)}} \cdot u_{j_{i}}^{*}\right\|_{U^{*}} \\
& \leq 2 B R K_{1}\left\|w^{*}\right\|_{W^{(*)}} .
\end{aligned}
$$

This shows that $\|\cdot\|_{W^{(*)}}$ and $\|\cdot\|_{Y^{*}}$ are equivalent on $\mathrm{c}_{00}\left(\oplus_{i} E_{k_{i}}^{*}\right)$. It is easy to verify that $\sum_{i} P_{k_{i}}^{E^{*}}$, which defines a norm-1 projection of $W^{(*)}$ onto $\overline{\bigoplus_{i} E_{k_{i}}^{*}}$, is also a norm-1 projection of $Y^{*}$ onto $\overline{\bigoplus_{i} E_{k_{i}}^{*}}$. It follows that $\frac{1}{2 B R K_{1}}\|w\|_{W} \leq\|w\|_{Y} \leq\|w\|_{W}$ for all $w \in \mathrm{c}_{00}\left(\oplus_{i} E_{k_{i}}\right)$.

A very similar argument shows that $\|y\|_{Y} \leq\|y\|_{Z} \leq 2 B L K_{1}\|y\|_{Y}$ for all $y \in$ $\mathrm{c}_{00}\left(\oplus_{i} E_{k_{i}}\right)$. Indeed, the first inequality is clear from the definition of $\|\cdot\|_{Z}$, whereas the second one is obtained by a computation similar to the one in (25).

We have thus shown that the 2-embedding $T: \tilde{X} \rightarrow W$ becomes a $8 B^{2} L R K_{1}^{2}$ embedding viewed as a map $\tilde{X} \rightarrow Z$. Hence $X K$-embeds into $Z$, where $K=$ $8 B^{2} L R K_{1}^{2} K_{2}$.

We conclude this paper with two applications of our embedding theorems. The first one is the observation that our results here give an alternative proof to the main theorem in [1].

Theorem 22 ([1]). Let $X$ be a separable, reflexive Banach space and let $1 \leq q \leq$ $p \leq \infty$. The following are equivalent.

(a) $X$ satisfies $(p, q)$ tree estimates.

(b) $X$ is isomorphic to a subspace of a reflexive space $Z$ having an FDD which satisfies $(p, q)$ estimates.

(c) $X$ is isomorphic to a quotient of a reflexive space $Z$ having an FDD which satisfies $(p, q)$ estimates.

Here an FDD $\left(E_{n}\right)$ of a Banach space $Z$ is said to satisfy $(p, q)$ estimates if there is a constant $C>0$ such that for every block sequence $\left(x_{i}\right)$ of $\left(E_{n}\right)$ we have

$$
C^{-1}\left(\sum\left\|x_{i}\right\|^{p}\right)^{1 / p} \leq\left\|\sum x_{i}\right\| \leq C\left(\sum\left\|x_{i}\right\|^{q}\right)^{1 / q}
$$

and a Banach space $X$ is said to satisfy $(p, q)$ estimates if every normalized, weakly null tree $\left(x_{\alpha}\right)_{\alpha \in T_{\infty}}$ in $X$ has a branch that dominates the unit vector basis of $\ell_{p}$ and that is dominated by the unit vector basis of $\ell_{q}$. The family $\left(x_{\alpha}\right)_{\alpha \in T_{\infty}}$ in $X$ is called a normalized, weakly null tree if for all $\alpha \in T_{\infty} \cup\{\emptyset\}$ the sequence $\left(x_{(\alpha, n)}\right)$ is 
normalized and weakly null, and a branch of $\left(x_{\alpha}\right)_{\alpha \in T_{\infty}}$ is a sequence $\left(x_{\left(n_{1}, n_{2}, \ldots, n_{i}\right)}\right)$, where $n_{1}<n_{2}<\ldots$

The second, and main, application concerns the existence of universal spaces for the classes $C_{\alpha}$ defined in the Introduction. Recall that for each countable ordinal $\alpha$ the class $C_{\alpha}$ consists of all separable, reflexive spaces $X$ such that both $X$ and its dual $X^{*}$ have Szlenk index at most $\alpha$. Szlenk introduced his index to show that there is no separable, reflexive space that contains isomorphic copies of every separable, reflexive space [17.

The Szlenk index $\mathrm{Sz}(\cdot)$ has the following properties [17: for a separable space $X$, $\mathrm{Sz}(X)<\omega_{1}$ if and only if $X^{*}$ is separable (so $\bigcup_{\alpha<\omega_{1}} C_{\alpha}$ is the class of all separable, reflexive spaces); if $Y$ embeds into $X$, then $\mathrm{Sz}(Y) \leq \mathrm{Sz}(X)$; for all $\alpha<\omega_{1}$ there exists a separable, reflexive space $X$ such that $\mathrm{Sz}(X)>\alpha$. From these properties it follows immediately that if a separable space $Z$ contains isomorphic copies of every separable, reflexive space, then $Z^{*}$ is not separable, and so $Z$ cannot be reflexive. (Later J. Bourgain showed that such a space $Z$ must contain $C[0,1]$, and hence all separable Banach spaces [1.)

It seems natural to ask if there is, for each countable ordinal $\alpha$, a separable, reflexive space that is universal for $C_{\alpha}$. This question was indeed raised by Pełczyński motivated by the results of [1], which imply an affirmative answer for $\alpha=\omega$. In [13] we show that Pełczyński's question has an affirmative answer for all $\alpha<\omega_{1}$.

Theorem 23. For each countable ordinal $\alpha$ there is a separable, reflexive space $Z$ which is universal for the class $C_{\alpha}$.

This is a simplified version of our result which also includes estimates on embedding constants and determines the class $C_{\beta}$ in which the universal space $Z$ lives. The proof, which is given in [13, splits into two parts. We first prove that if $X \in C_{\alpha}$, then there exists $\gamma<\alpha$ such that $X$ satisfies subsequential $\left(\left(T_{\gamma, \frac{1}{2}}\right)^{*}, T_{\gamma, \frac{1}{2}}\right)$ tree estimates, where $T_{\gamma, \frac{1}{2}}$ is the Tsirelson space of order $\gamma$. The ingredient for the second part of the proof is Theorem 21 from this paper. We fix a sequence $\left(\alpha_{n}\right)$ of ordinals with $\alpha=\sup _{n}\left(\alpha_{n}+1\right)$, and for each $n \in \mathbb{N}$ we let $Z_{n}$ be a separable, reflexive space which is universal for the class $\mathcal{A}_{\left(\left(T_{\alpha_{n}, \frac{1}{2}}\right)^{*}, T_{\alpha_{n}, \frac{1}{2}}\right)}$. The $\ell_{2}$-direct sum $Z$ of the sequence $\left(Z_{n}\right)$ is then the required universal space for $C_{\alpha}$.

\section{REFERENCES}

[1] J. Bourgain, 'On separable Banach spaces, universal for all separable reflexive spaces', Proc. Amer. Math. Soc. 79 (1980) no. 2 241-246.

[2] S. F. Bellenot, R. Haydon, E. Odell, 'Quasi-reflexive and tree spaces constructed in the spirit of R. C. James', Banach space theory (Iowa City, IA, 1987), 19-43, Contemp. Math., 85, Amer. Math. Soc., Providence, RI, 1989.

[3] P. Dodos, V. Ferenczi, 'Some strongly bounded classes of Banach spaces', preprint.

[4] D. Gale and F.M. Stewart, 'Infinite games with perfect information', Contributions to the theory of games, Annals of Math. Studies no. 28 Princeton University Press (1953) 245-266.

[5] W. T. Gowers, 'An infinite Ramsey theorem and some Banach-space dichotomies', Ann. of Math. (2) 156 (2002) no. 3 797-833.

[6] W. B. Johnson, 'On quotients of $L_{p}$ which are quotients of $l_{p}$ ', Compositio Math. 34 (1977) no. $169-89$.

[7] W. B. Johnson and M. Zippin, 'On subspaces of quotients of $\left(\sum G_{n}\right)_{l_{p}}$ and $\left(\sum G_{n}\right)_{c_{0}}$ ', Proceedings of the International Symposium on Partial Differential Equations and the Geometry of Normed Linear Spaces, Jerusalem, 1972, Israel J. Math. 13 (1972) 311-316.

[8] D. A. Martin, 'Borel determinacy', Ann. of Math. (2) 102 (1975) no. 2 363-371.

[9] B. Maurey, V. D. Milman and N. Tomczak-Jaegermann, 'Asymptotic infinite-dimensional theory of Banach spaces', Oper. Theory: Adv. Appl. 77 (1994) 149-175.

[10] E. Odell and Th. Schlumprecht, 'Trees and branches in Banach spaces', Trans. Amer. Math. Soc. 354 (2002) no. 10 4085-4108.

[11] E. Odell and Th. Schlumprecht, 'A universal reflexive space for the class of uniformly convex Banach spaces', Mathematische Annalen (to appear). 
[12] E. Odell, Th. Schlumprecht and A. Zsák, 'On the structure of asymptotic $\ell_{p}$ spaces', submitted to LMS.

[13] E. Odell, Th. Schlumprecht and A. Zsák, 'Banach spaces with bounded Szlenk index', preprint.

[14] C. Rosendal, 'Infinite asymptotic games', preprint.

[15] H. P. Rosenthal, 'Block-determined finite-dimensional decompositions', preprint.

[16] G. Schechtman, 'On Pełczyński's paper "Universal bases"', Israel J. Math. 22 (1975) no. 3-4 181-184.

[17] W. Szlenk, 'The non-existence of a separable reflexive Banach space universal for all separable reflexive Banach spaces', Studia Math. 301968 53-61.

[18] M. Zippin, 'Banach spaces with separable duals', Trans. Amer. Math. Soc. 310 no. 1 (1988) $371-379$.

Department of Mathematics, The University of Texas, 1 University Station C1200, Austin, TX 78712, USA

E-mail address: odell@math.utexas.edu

Department of Mathematics, Texas A\&M University, College Station, TX 78712 , USA

E-mail address: thomas.schlumprecht@math.tamu.edu

School of Mathematical Sciences, University of Nottingham, University Park, NotTINGHAM NG7 2RD, United KingDOM

E-mail address: andras.zsak@maths.nottingham.ac.uk 